PHYSICAL REVIEW D 90, 123515 (2014)

\title{
Regularized cosmological power spectrum and correlation function in modified gravity models
}

\author{
Atsushi Taruya \\ Yukawa Institute for Theoretical Physics, Kyoto University, Kyoto 606-8502, Japan \\ and Kavli Institute for the Physics and Mathematics of the Universe (Kavli IPMU, WPI), Todai Institutes \\ for Advanced Study, The University of Tokyo, Kashiwa, Chiba 277-8583, Japan \\ Takahiro Nishimichi and Francis Bernardeau \\ Institut d'Astrophysique de Paris, CNRS UMR 7095 and UPMC, 98bis, bd Arago, F-75014 Paris, France \\ Takashi Hiramatsu \\ Yukawa Institute for Theoretical Physics, Kyoto University, Kyoto 606-8502, Japan \\ Kazuya Koyama \\ Institute of Cosmology \& Gravitation, University of Portsmouth, Dennis Sciama Building, \\ Portsmouth PO1 3FX, United Kingdom
}

(Received 29 August 2014; published 12 December 2014)

\begin{abstract}
Based on the multipoint propagator expansion, we present resummed perturbative calculations for cosmological power spectra and correlation functions in the context of modified gravity. In a wide class of modified gravity models that have a screening mechanism to recover general relativity (GR) on small scales, we apply the eikonal approximation to derive the governing equation for resummed propagator that partly includes the nonperturbative effect in the high- $k$ limit. The resultant propagator in the high- $k$ limit contains the new corrections arising from the screening mechanism as well as the standard exponential damping. We explicitly derive the expression for new high- $k$ contributions in specific modified gravity models, and find that in the case of $f(R)$ gravity for a currently constrained model parameter, the corrections are basically of the subleading order and can be neglected. Thus, in $f(R)$ gravity, similarly to the GR case, we can analytically construct the regularized propagator that reproduces both the resummed high- $k$ behavior and the low- $k$ results computed with standard perturbation theory, consistently taking account of the nonlinear modification of gravity valid at large scales. With the regularized multipoint propagators, we give predictions for power spectrum and correlation function at one-loop order, and compare those with $N$-body simulations in $f(R)$ gravity model. As an important application, we also discuss the redshift-space distortions and compute the anisotropic power spectra and correlation functions.
\end{abstract}

DOI: $10.1103 /$ PhysRevD.90.123515

PACS numbers: 98.80.-k, 98.62.Py, 98.65.-r

\section{INTRODUCTION}

The precision observation of large-scale structure of the Universe now plays a very crucial role in scrutinizing the standard cosmological model that has emerged recently based on the multiple cosmological observations. Amongst various cosmological issues, one important subject is to clarify the origin and nature of cosmic acceleration, first discovered by the distant supernova observations $[1,2]$. The cosmic acceleration may be originated from the dark energy, or rather it may indicate the breakdown of general relativity on very large scales. To observationally explore this, the measurements of both the cosmic expansion and the growth of structure are thought to be essential, giving us a chance to test gravity on cosmological scales or to constrain dark energy equation of state (e.g., [3] for review). The large-scale structure observations with galaxy redshift surveys indeed offer an opportunity to measure these two quantities simultaneously.

The key measurements are the baryon acoustic oscillations (BAO) and redshift-space distortions (RSD), imprinted on the large-scale clustering pattern of galaxy distribution. With $\mathrm{BAO}$ as a standard ruler, we can simultaneously measure the angular diameter distance and Hubble parameter at the distant galaxies through the Alcock-Paczynski effect (e.g., [4-9]). On the other hand, RSD caused by the peculiar velocity of galaxies induces apparent clustering anisotropies, whose strength is related to the growth rate of structure formation (e.g., [10-13]). Since both BAO and RSD are now reliably and simultaneously measured through the clustering statistics of galaxy distribution (e.g., [14-18] for recent measurements) typically on scales close to the linear regime of gravitational evolution, the precision estimation of power spectrum and/ 
or correlation function is a major priority of the ongoing and upcoming galaxy surveys.

With increasing interests in precision measurements, accurate theoretical modelings of power spectrum and/or correlation function is crucial and is essential to correctly estimate the geometric distances and structure growth, taking full account of the nonlinear systematics including gravitational clustering and RSD. Development of theoretical templates is thus an important research subject, and there have been numerous numerical and analytical studies along this line [19-38]. Thanks to these efforts, we are now able to discuss the accuracy of theoretical template at a percent level. However, one important remark is that the calculation of such templates, especially for the prediction of gravitational clustering, heavily relies on the underlying theory of gravity. So far, general relativity (GR) has been implicitly assumed as the underlying theory of gravity in most studies. As a consequence, although such templates can be employed for consistency tests of GR, their use for characterizing or detecting deviation from GR gravity can be limited.

Further theoretical developments are therefore required in a wide context of modified gravity models. While a model-independent approach, in which we do not assume gravity but rather parametrize it in a fairly generic way (e.g., [39-43]) is very helpful and should be exploited, most of the approaches proposed so far have been restricted to the linear regime. Since the applicable range of linear theory calculation is known to be rather narrower at lower redshifts, our ability to constrain or test such models is expected to be significantly reduced [44].

In this paper, we attempt to extend the framework of theoretical templates to deal with modified theories of gravity. Here, we specifically examine this issue based on the analytical approach with perturbation theory calculations, relevant for the measurement of BAO and RSD on large scales. Previously, we have presented the basic formalism to treat general modified gravity models [45], and in specific gravity models, we have computed power spectra in both real and redshift spaces based on the standard perturbation theory (PT) [46] (see also Refs. $[47,48]$ for related works). The standard PT is, however, known to produce a poorly convergent series expansion, and because of the bad high- $k$ behavior (e.g., $[25,49,50])$, difficulty arises in computing the correlation function through a direct integration of power spectrum.

In the present paper, we shall apply the specific resummed PT scheme referred to as the multipoint propagator expansion or $\Gamma$ expansion [26]. The building blocks of this PT scheme are the multipoint propagators, with which the nonperturbative properties at high- $k$ can be efficiently resummed, giving us an improved convergence of the PT expansion. In the case of GR, making full use of the analytical properties, the regularized propagators, which consistently reproduces both the standard PT results at low- $k$ and the expected resummed behaviors at high- $k$, have been successfully constructed [51,52], and the $\Gamma$ expansion has been applied to the predictions of realand redshift-space power spectra and correlation functions, showing a very good agreement with $\mathrm{N}$-body simulations $[29,53,54]$. Clearly, a crucial point for applying this approach to the modified gravity models is whether we can systematically construct regularized propagators in a semianalytic manner. Here, we specifically show that while there appear nontrivial corrections originating from the screening mechanism in modified gravity model, in the case of $f(R)$ gravity model for a currently constrained model parameter, these corrections are basically small. Thus, in $f(R)$ gravity, the propagator can be constructed in a similar manner to the GR case. Then the analytically computed propagators are compared with $\mathrm{N}$-body simulations, and a good agreement is found. With these propagators as building blocks, we will proceed to the calculation of the power spectrum and correlation function in both real and redshift spaces.

The paper is organized as follows. In Sec. II, we briefly review the basic formalism to treat perturbations in general modified gravity models, and introduce a resummed PT scheme based on the multipoint propagator expansion. Section III discusses the nonperturbative high- $k$ behavior of the propagators based on the eikonal approximation, and Sec. IV presents an explicit expression for matter power spectrum in terms of the regularized propagators, which satisfy both the expected high- $k$ and low- $k$ behaviors. Then, the comparison of PT results with $N$-body simulations is made in Sec. V, and the applications to the redshift-space observables are discussed in Sec. VI. Finally, the newly developed PT calculation is compared with standard PT prediction in Sec. VII, and we summarize our findings in Sec. VIII.

\section{BASIC EQUATIONS FOR PERTURBATIONS}

In this section, we begin by reviewing the framework to treat the evolution of matter fluctuations in modified gravity models [45], and present a set of basic equations relevant for the perturbation theory (PT) treatment. Then, a resummed PT scheme with multipoint propagator expansion [26] is briefly reviewed, and the properties of these multipoint propagators are mentioned.

\section{A. Dynamics of matter fluctuations in modified theories of gravity}

In this paper, we are particularly interested in the evolution of matter fluctuations, ignoring a tiny contribution of massive neutrinos. Inside the Hubble horizon, the so-called quasistatic approximation may be applied, and the time derivatives of the perturbed quantities can be neglected compared to the spatial derivatives. In GR, based on this approximation, we can find the Newtonian correspondence, 
and the standard Poisson equation is recovered. On the other hand, in modified theory of gravity, the Poisson equation is generically modified due to a new scalar degree of freedom, referred to as the scalaron. On large scales, the scalaron $\varphi$ mediates the scalar force, and behaves like the Brans-Dicke scalar field without potential and selfinteractions, while it should acquire some interaction terms on small scales, which play an important role to recover GR and to evade the solar-system constraints. Indeed, there are several known mechanism such as chameleon and Vainshtein mechanisms (e.g., [55,56]), in which the nonlinear interaction terms naturally arise and eventually become dominant, leading to a recovery of GR. As a result, the Poisson equation is coupled to the field equation for scalaron $\varphi$ with self-interaction term. Under the quasistatic approximation, we have [45] (see also Ref. [47])

$$
\begin{gathered}
\frac{1}{a} \nabla^{2} \psi=\frac{\kappa^{2}}{2} \rho_{\mathrm{m}} \delta-\frac{1}{2 a^{2}} \nabla^{2} \varphi \\
\left(3+2 \omega_{\mathrm{BD}}\right) \frac{1}{a^{2}} \nabla^{2} \varphi=-2 \kappa^{2} \rho_{\mathrm{m}} \delta-\mathcal{I}(\varphi)
\end{gathered}
$$

with $\kappa^{2}=8 \pi G$ and $\omega_{\mathrm{BD}}$ being the Brans-Dicke parameter. The quantities $\psi$ is the Newton potential, and the function $\mathcal{I}$ represents the nonlinear self-interaction, which may be generally expanded as

$$
\begin{aligned}
\mathcal{I}(\varphi)= & M_{1}(k) \\
& +\frac{1}{2} \int \frac{d^{3} \boldsymbol{k}_{1} d^{3} \boldsymbol{k}_{2}}{(2 \pi)^{3}} \delta_{\mathrm{D}}\left(\boldsymbol{k}-\boldsymbol{k}_{12}\right) M_{2}\left(\boldsymbol{k}_{1}, \boldsymbol{k}_{2}\right) \varphi\left(\boldsymbol{k}_{1}\right) \varphi\left(\boldsymbol{k}_{2}\right) \\
& +\frac{1}{3 !} \int \frac{d^{3} \boldsymbol{k}_{1} d^{3} \boldsymbol{k}_{2} d^{3} \boldsymbol{k}_{3}}{(2 \pi)^{6}} \delta_{\mathrm{D}}\left(\boldsymbol{k}-\boldsymbol{k}_{123}\right) \\
& \times M_{3}\left(\boldsymbol{k}_{1}, \boldsymbol{k}_{2}, \boldsymbol{k}_{3}\right) \varphi\left(\boldsymbol{k}_{1}\right) \varphi\left(\boldsymbol{k}_{2}\right) \varphi\left(\boldsymbol{k}_{3}\right)+\ldots
\end{aligned}
$$

On the other hand, for the matter sector, the evolution of matter fluctuations is governed by the conservation of energy momentum tensor, which would remain unchanged even if the gravity sector is modified. Under the singlestream approximation, which is relevant for the scale of our interest, the matter fluctuations are treated as a pressureless fluid flow, whose evolution equations are given by [46]

$$
\begin{gathered}
\frac{\partial \delta}{\partial t}+\frac{1}{a} \nabla \cdot[(1+\delta) \boldsymbol{v}]=0, \\
\frac{\partial \boldsymbol{v}}{\partial t}+H \boldsymbol{v}+\frac{1}{a}(\boldsymbol{v} \cdot \nabla) \cdot \boldsymbol{v}=-\frac{1}{a} \nabla \psi .
\end{gathered}
$$

Equations (2)-(4) are the basic equations for perturbations in a general framework of modified gravity models. In Fourier space, they can be reduced to a more compact form. Assuming the irrotationality of fluid quantities, the velocity field is expressed in terms of the velocity divergence, $\theta=\nabla \cdot v /(a H)$. Then, we introduce the two-component multiplet (e.g., [49]):

$$
\Psi_{a}(\boldsymbol{k} ; t)=(\delta(\boldsymbol{k} ; t),-\theta(\boldsymbol{k} ; t)),
$$

where the subscript $a=1,2$ selects the density and the velocity components of CDM plus baryons. The governing equations for $\Psi_{a}$ become [45]

$$
\begin{aligned}
& \frac{\partial \Psi_{a}(\boldsymbol{k} ; \tau)}{\partial \tau}+\boldsymbol{\Omega}_{a b}(k ; \tau) \Psi_{b}(\boldsymbol{k} ; \tau) \\
& =\int \frac{d^{3} \boldsymbol{k}_{1} d^{3} \boldsymbol{k}_{2}}{(2 \pi)^{3}} \delta_{\mathrm{D}}\left(\boldsymbol{k}-\boldsymbol{k}_{12}\right) \gamma_{a b c}\left(\boldsymbol{k}_{1}, \boldsymbol{k}_{2}\right) \Psi_{b}\left(\boldsymbol{k}_{1} ; \tau\right) \Psi_{c}\left(\boldsymbol{k}_{2} ; \tau\right) \\
& \quad+\delta_{a 2} \sum_{n=2} \int \frac{d^{3} \boldsymbol{k}_{1} \cdots d^{3} \boldsymbol{k}_{n}}{(2 \pi)^{3(n-1)}} \\
& \quad \times \delta_{\mathrm{D}}\left(\boldsymbol{k}-\boldsymbol{k}_{1 \cdots n}\right) \sigma^{(n)}\left(\boldsymbol{k}_{1}, \ldots, \boldsymbol{k}_{n} ; \tau\right) \Psi_{1}\left(\boldsymbol{k}_{1} ; \tau\right) \cdots \Psi_{1}\left(\boldsymbol{k}_{n} ; \tau\right),
\end{aligned}
$$

where the time variable $\tau$ is defined by $\tau=\ln a(t)$, and $\delta_{a b}$ is the Kronecker delta. Here, we introduced the shortcut notations, $\boldsymbol{k}_{12}=\boldsymbol{k}_{1}+\boldsymbol{k}_{2}$ and $\boldsymbol{k}_{1 \cdots n}=\boldsymbol{k}_{1}+\cdots+\boldsymbol{k}_{n}$. The matrix $\Omega_{a b}$ is given by

$$
\Omega_{a b}(k ; \tau)=\left(\begin{array}{cc}
0 & -1 \\
-\frac{\kappa^{2}}{2} \frac{\rho_{\mathrm{m}}}{H^{2}}\left[1+\frac{1}{3} \frac{(k / a)^{2}}{\Pi(k)}\right] & 2+\frac{\dot{H}}{H^{2}}
\end{array}\right)
$$

with the function $\Pi$ defined by

$$
\Pi(k)=\frac{1}{3}\left\{\left(3+2 \omega_{\mathrm{BD}}\right) \frac{k^{2}}{a^{2}}+M_{1}\right\} .
$$

From the $(2,1)$ component of $\Omega_{a b}$, we can define the effective Newton constant as

$$
G_{\mathrm{eff}}=G\left[1+\frac{1}{3} \frac{(k / a)^{2}}{\Pi(k)}\right]
$$

Note that in the cases with $M_{1}=0$, the effective Newton constant is given by

$$
G_{\mathrm{eff}}=\frac{2\left(2+\omega_{\mathrm{BD}}\right)}{3+2 \omega_{\mathrm{BD}}} G .
$$

For a positive $\omega_{\mathrm{BD}}>0$, the effective gravitational constant is larger than GR and the gravitational force is enhanced. On the other hand, if $M_{1} \gg k^{2} / a^{2}, G_{\text {eff }}$ becomes $G$.

In Eq. (7), there appear two types of vertex functions. One is the standard vertex function arising from the nonlinearity of the fluid flow, $\gamma_{a b c}$ : 


$$
\gamma_{a b c}\left(\boldsymbol{k}_{1}, \boldsymbol{k}_{2}\right)= \begin{cases}\frac{1}{2}\left\{1+\frac{\boldsymbol{k}_{1} \cdot \boldsymbol{k}_{2}}{\left|\boldsymbol{k}_{2}\right|^{2}}\right\} & ;(a, b, c)=(1,1,2), \\ \frac{1}{2}\left\{1+\frac{\boldsymbol{k}_{2} \cdot \boldsymbol{k}_{1}}{\left|\boldsymbol{k}_{1}\right|^{2}}\right\} & ;(a, b, c)=(1,2,1), \\ \frac{\left(\boldsymbol{k}_{1} \cdot \boldsymbol{k}_{2}\right)\left|\boldsymbol{k}_{1}+\boldsymbol{k}_{2}\right|^{2}}{2\left|\boldsymbol{k}_{1}\right|^{2}\left|\boldsymbol{k}_{2}\right|^{2}} & ;(a, b, c)=(2,2,2), \\ 0 & ; \text { otherwise. }\end{cases}
$$

Note the symmetric properties of the vertex function, $\gamma_{a b c}\left(\boldsymbol{k}_{1}, \boldsymbol{k}_{2}\right)=\gamma_{a c b}\left(\boldsymbol{k}_{2}, \boldsymbol{k}_{1}\right)$. Another vertex function is characterized by the kernel $\sigma^{(n)}$, which represents the mode coupling of the density fields $\Psi_{1}$ with velocity-divergence field. This coupling comes from the nonlinear interaction terms of the scalaron $\varphi$ [i.e., Eqs. (1) and (2) through (3)]. The explicit form of the higher-order vertex functions is given by (see Appendix B for derivation):

$$
\begin{aligned}
& \sigma^{(2)}\left(\boldsymbol{k}_{1}, \boldsymbol{k}_{2} ; \tau\right)=-\frac{1}{12 H^{2}}\left(\frac{\kappa^{2} \rho_{\mathrm{m}}}{3}\right)^{2}\left(\frac{k_{12}^{2}}{a^{2}}\right) \frac{M_{2}\left(\boldsymbol{k}_{1}, \boldsymbol{k}_{2}\right)}{\Pi\left(k_{12}\right) \Pi\left(k_{1}\right) \Pi\left(k_{2}\right)}, \\
& \sigma^{(3)}\left(\boldsymbol{k}_{1}, \boldsymbol{k}_{2}, \boldsymbol{k}_{3} ; \tau\right)=-\frac{1}{36 H^{2}}\left(\frac{\kappa^{2} \rho_{\mathrm{m}}}{3}\right)^{3}\left(\frac{k_{123}^{2}}{a^{2}}\right) \frac{1}{\Pi\left(k_{123}\right) \Pi\left(k_{1}\right) \Pi\left(k_{2}\right) \Pi\left(k_{3}\right)}\left\{M_{3}\left(\boldsymbol{k}_{1}, \boldsymbol{k}_{2}, \boldsymbol{k}_{3}\right)-\frac{M_{2}\left(\boldsymbol{k}_{12}, \boldsymbol{k}_{3}\right) M_{2}\left(\boldsymbol{k}_{1}, \boldsymbol{k}_{2}\right)}{\Pi\left(k_{12}\right)}\right\}, \\
& \sigma^{(4)}\left(\boldsymbol{k}_{1}, \boldsymbol{k}_{2}, \boldsymbol{k}_{3}, \boldsymbol{k}_{4} ; \tau\right)=- \frac{1}{144 H^{2}}\left(\frac{\kappa^{2} \rho_{\mathrm{m}}}{3}\right)^{4}\left(\frac{k_{1234}^{2}}{a^{2}}\right) \frac{1}{\Pi\left(k_{1234}\right) \Pi\left(k_{1}\right) \Pi\left(k_{2}\right) \Pi\left(k_{3}\right) \Pi\left(k_{4}\right)} \\
& \times {\left[M_{4}\left(\boldsymbol{k}_{1}, \boldsymbol{k}_{2}, \boldsymbol{k}_{3}, \boldsymbol{k}_{4}\right)+\frac{1}{3}\left\{\frac{M_{2}\left(\boldsymbol{k}_{1}, \boldsymbol{k}_{2}\right)}{\Pi\left(k_{12}\right)}\left(\frac{M_{2}\left(\boldsymbol{k}_{12}, \boldsymbol{k}_{34}\right) M_{2}\left(\boldsymbol{k}_{3}, \boldsymbol{k}_{4}\right)}{\Pi\left(k_{34}\right)}-6 M_{3}\left(\boldsymbol{k}_{12}, \boldsymbol{k}_{2}, \boldsymbol{k}_{3}\right)\right)\right.\right.} \\
&+\left.\left.2 \frac{M_{2}\left(\boldsymbol{k}_{123}, \boldsymbol{k}_{4}\right)}{\Pi\left(k_{123}\right)}\left(\frac{M_{2}\left(\boldsymbol{k}_{12}, \boldsymbol{k}_{13}\right) M_{2}\left(\boldsymbol{k}_{1}, \boldsymbol{k}_{2}\right)}{\Pi\left(k_{12}\right)}-M_{3}\left(\boldsymbol{k}_{1}, \boldsymbol{k}_{2}, \boldsymbol{k}_{3}\right)\right)\right\}\right] .
\end{aligned}
$$

Note that the expression of vertex functions $\sigma^{(3)}$ and $\sigma^{(4)}$ is not yet symmetrized under the permutation of wave vectors, and it has to be symmetrized.

So far the framework to treat perturbations is general, and can be applied to any gravity model that satisfies the conservation law of the matter sector. As representative examples of modified gravity models that can explain the late-time cosmic acceleration, we shall below consider the $f(R)$ gravity [57,58] and Dvali-Gabadadze-Poratti (DGP) model [59], and present the explicit expressions for modeldependent parameters $\omega_{\mathrm{BD}}$ and coupling functions $\Pi$ and $M_{i}$. While these models are rather specific and have been tightly constrained recently by observations, the mechanisms to recover GR on small scales are typical and a broad class of modified gravity models can fall into either of two models. We thus expect that even the PT calculations in these specific modified gravity models can give a fairly generic view on the deviation of gravity from GR.

\section{1. $f(R)$ gravity}

The $f(R)$ gravity is a representative modified gravity model for which the Einstein-Hilbert action is generalized to include an arbitrary function of the scalar curvature $R$ :

$$
S=\int d^{4} x \sqrt{-g}\left[\frac{R+f(R)}{2 \kappa^{2}}\right]+L_{\mathrm{m}},
$$

where $L_{\mathrm{m}}$ is the Lagrangian for matter sector. This theory is known to be equivalent to the Brans-Dicke theory with parameter $\omega_{\mathrm{BD}}=0$, but due to the nonlinear functional form of $R$, the Brans-Dicke scalar can acquire a nontrivial potential. This can be seen from the trace of the modified Einstein equations. In the universe dominated by ordinary matter, we have

$$
3 \square f_{R}-R+f_{R} R-2 f=-\kappa^{2} \rho_{\mathrm{m}}
$$

where $f_{R}=d f / d R$ and $\square=\nabla_{\mu} \nabla^{\mu}$. The field $f_{R}$ is identified with the scalaron, i.e., the extra scalar field, and its perturbations are defined as

$$
\varphi=\delta f_{R} \equiv f_{R}-\bar{f}_{R},
$$

where the bar indicates that the quantity is evaluated on the background universe. Imposing the conditions $\left|\bar{f}_{R}\right| \ll 1$ and $|\bar{f} / \bar{R}| \ll 1$, the background expansion can be close to $\Lambda \mathrm{CDM}$ cosmology, and the quasistatic approximation leads to

$$
3 \frac{1}{a^{2}} \nabla^{2} \varphi=-\kappa^{2} \rho_{\mathrm{m}} \delta+\delta R, \quad \delta R \equiv R\left(f_{R}\right)-R\left(\bar{f}_{R}\right),
$$

The above equation indeed corresponds to Eq. (2) with $\omega_{\mathrm{BD}}=0$. Then, expanding $\delta R$ in terms of $\varphi$, we obtain the explicit functional form of the coupling functions: 


$$
M_{n}(\tau)=\frac{d^{n} \bar{R}\left(f_{R}\right)}{d f_{R}^{n}}
$$

which only depends on time. Then, this gives

$$
\Pi(k)=\left(\frac{k}{a}\right)^{2}+\frac{\bar{R}_{, f}}{3},
$$

where we define $\bar{R}_{f}(\tau) \equiv d \bar{R}\left(f_{R}\right) / d f_{R}$.

In this paper, we will present the results of PT calculations in $f(R)$ gravity, and the predictions of propagator, power spectrum, and correlation function are compared with $N$-body simulations. For this purpose, in this paper, we will below consider the specific function of the form:

$$
f(R) \propto \frac{R}{A R+1},
$$

where $A$ is a dimensional constant of length squared. In particular, we are interested in the high curvature limit $A R \gg 1$, and $f(R)$ can be expanded as

$$
f(R) \simeq-2 \kappa^{2} \rho_{\Lambda}+\left|f_{R 0}\right| \frac{\bar{R}_{0}^{2}}{R} .
$$

Here, $\rho_{\Lambda}$ is the constant energy density related to $A$. The quantity $\bar{R}_{0}$ is the background curvature at present time, and we defined $f_{R 0}=\bar{f}_{R}\left(\bar{R}_{0}\right)$. With the current observational constraint $\left|f_{R 0}\right| \ll 1$ (e.g., [60-64], see also [65] for a strong constraint from small-scales), the background cosmology becomes indistinguishable with $\Lambda \mathrm{CDM}$ model, but the extra term is still non-negligible for the evolution of matter fluctuations, giving rise to a different growth history of structure.

\section{DGP model}

The DGP braneworld model is another modified gravity model that has a screening mechanism. The DGP model is the $5 \mathrm{D}$ gravity theory with the induced $4 \mathrm{D}$ gravity on a brane in which we are living. Thus, on large scales larger than the characteristic scale $r_{c}$, the gravity becomes $5 D$, while on small scales, gravity becomes $4 D$, but it is not described by GR. As a result, the Friedman equation is modified on the brane [59]:

$$
\epsilon \frac{H}{r_{c}}=H^{2}-\frac{\kappa^{2} \rho_{\mathrm{m}}}{3},
$$

where $\epsilon= \pm 1$ represents two distinct branches of the solutions ( $\epsilon=1$ is the self-accelerating branch, and -1 is called the normal branch).

Notable point in the DGP model is that the GR is recovered via the Vainshtein mechanism, by which the scalaron becomes massless, but acquires a large secondorder derivative interaction. The resultant coupling functions become [45]

$$
\begin{aligned}
& M_{1}=0, \quad M_{2}\left(\boldsymbol{k}_{1}, \boldsymbol{k}_{2} ; \tau\right)=2 \frac{r_{c}^{2}}{a^{4}}\left\{k_{1}^{2} k_{2}^{2}-\left(\boldsymbol{k}_{1} \cdot \boldsymbol{k}_{2}\right)^{2}\right\}, \\
& M_{i}=0 \quad(i \geq 3) .
\end{aligned}
$$

The quasistatic perturbations on 4D brane are described by the Brans-Dicke theory, where the Brans-Dicke parameter is given by

$\omega_{\mathrm{BD}}(\tau)=\frac{3}{2}\{\beta(\tau)-1\} ; \beta(\tau)=1-2 \epsilon H r_{c}\left(1+\frac{\dot{H}}{3 H^{2}}\right)$,

with $\dot{H}$ being the cosmic time derivative of the Hubble parameter. Then, the function $\Pi$ becomes

$$
\Pi(k)=\beta(\tau)\left(\frac{k}{a}\right)^{2} .
$$

\section{B. Multipoint propagator expansion}

Provided the basic equations for matter fluctuations, a straightforward approach to deal with the nonlinear evolution perturbatively is to just expand the perturbed quantities like $\Psi_{a}=\Psi_{a}^{(1)}+\Psi_{a}^{(2)}+\ldots$, and to solve the equations order by order, regarding the initial field as a small expansion parameter. This is the so-called standard PT treatment [46]. As we mentioned in Sec. I, the standard PT is known to produce a poorly convergent series expansion, and is difficult to compute the correlation function because of the bad UV behavior.

Alternatively, we may first introduce the nonperturbative statistical quantities, and expand the statistical quantities for our interest in terms of these. The multipoint propagator expansion or the $\Gamma$ expansion is one such PT expansion, and is regarded as a resummed PT treatment, in which the standard PT expansion is reorganized by the nonperturbative quantities [26]. A key property is that all the statistical quantities such as the power spectra and bispectra can be reconstructed by an expansion series written solely in terms of the multipoint propagators. The multi-point propagator is a fully nonperturbative quantity, and with this object, a good convergence of the PT expansion is guaranteed. This is in marked contrast to the standard PT expansion. Although these have been confirmed and checked in the case of GR, we expect them to hold even in modified gravity models as long as the deviation from GR is small.

The $(p+1)$-point propagator is defined by

$$
\begin{aligned}
& \frac{1}{p !}\left\langle\frac{\delta^{p} \Psi_{a}(\boldsymbol{k} ; \tau)}{\delta \delta_{0}\left(\boldsymbol{k}_{1}\right) \cdots \delta \delta_{0}\left(\boldsymbol{k}_{p}\right)}\right\rangle \\
& \quad=\delta_{D}\left(\boldsymbol{k}-\boldsymbol{k}_{1 \cdots p}\right) \frac{1}{(2 \pi)^{3(p-1)}} \Gamma_{a}^{(p)}\left(\boldsymbol{k}_{1}, \ldots, \boldsymbol{k}_{p} ; \tau\right),
\end{aligned}
$$

with $\langle\cdots\rangle$ being the ensemble average. Here, $\delta_{0}$ is the initial density field given at an early time $\tau_{0}$. With the multi-point 
propagator, the power spectra of cosmic fields are systematically constructed as follows. Defining the power spectra $P_{a b}$ as

$$
\left\langle\Psi_{a}(\boldsymbol{k} ; \tau) \Psi_{b}\left(\boldsymbol{k}^{\prime} ; \tau\right)\right\rangle=(2 \pi)^{3} \delta_{\mathrm{D}}\left(\boldsymbol{k}+\boldsymbol{k}^{\prime}\right) P_{a b}(|\boldsymbol{k}| ; \tau),
$$

we have [26]

$$
\begin{aligned}
P_{a b}(k ; \tau)= & \Gamma_{a}^{(1)}(k ; \tau) \Gamma_{b}^{(1)}(k ; \tau) P_{0}(k) \\
& +\sum_{n=2} n ! \int \frac{d^{3} \boldsymbol{q}_{1} \cdots d^{3} \boldsymbol{q}_{n}}{(2 \pi)^{3(n-1)}} \delta_{\mathrm{D}}\left(\boldsymbol{k}-\boldsymbol{q}_{1 \cdots n}\right) \\
& \times \Gamma_{a}^{(n)}\left(\boldsymbol{q}_{1}, \ldots, \boldsymbol{q}_{n} ; \tau\right) \Gamma_{b}^{(n)}\left(\boldsymbol{q}_{1}, \cdots, \boldsymbol{q}_{n} ; \tau\right) \\
& \times P_{0}\left(q_{1}\right) \cdots P_{0}\left(q_{n}\right),
\end{aligned}
$$

where the quantity $P_{0}$ is the initial power spectrum defined as

$$
\left\langle\delta_{0}(\boldsymbol{k}) \delta_{0}\left(\boldsymbol{k}^{\prime}\right)\right\rangle=(2 \pi)^{3} \delta_{\mathrm{D}}\left(\boldsymbol{k}+\boldsymbol{k}^{\prime}\right) P_{0}(k) .
$$

As it is clear from Eq. (30), the nonlinear effects in the power spectrum are wholly encapsulated in the multipoint propagators, and thus the construction of the propagators keeping their nonperturbative properties is quite essential in the analytic treatment of PT. Therefore, subsequent sections are devoted to the discussion on how to analytically construct the propagators in the context of modified gravity. Section III discusses the nonperturbative high- $k$ behavior of the propagators based on the eikonal approximation, and Sec. IV presents a consistent construction of the regularized propagators which satisfies both the expected high- $k$ and low- $k$ behaviors.

\section{RESUMMED LINEAR PROPAGATOR WITH EIKONAL APPROXIMATION}

In this section, we derive the resummed linear propagator, in which the behaviors of the high- $k$ limit is reproduced at the tree-level calculation as a result of resummation. This resummed linear propagator will be used to systematically construct the multipoint propagator in next section. Here, following Ref. [66], we apply the eikonal approximation to the perturbation equations (7). The eikonal approximation enables us to derive the effective evolution equation for short-wave fluctuations under the influence of long-wave modes, which are regarded as external random background. With this treatment, if we neglect the nonlinear mode couplings, the fluid equations can be rewritten as linear equations embedded in an external random medium.

\section{A. Eikonal approximation}

To be more explicit, consider first the nonlinear mode coupling in Eq. (7) associated with standard vertex function, $\gamma_{a b c}\left(\boldsymbol{k}_{1}, \boldsymbol{k}_{2}\right)$. Through the relation $\boldsymbol{k}=\boldsymbol{k}_{1}+\boldsymbol{k}_{2}$, the contribution of the nonlinear coupling can be split into two different cases: the one coming from coupling two modes of very different amplitudes, $k_{1} \ll k_{2}$ or $k_{2} \ll k_{1}$, and the one coming from coupling two modes of comparable amplitudes. In the first case, the small wave modes ought to be much smaller than $\boldsymbol{k}$. Let us denote these small modes by $\boldsymbol{q}$, and divide the domain of integral into soft and hard domains. Then, the coupling term may be rewritten as [66]

$$
\begin{gathered}
\int \frac{d^{3} \boldsymbol{k}_{1} d^{3} \boldsymbol{k}_{2}}{(2 \pi)^{3}} \delta_{\mathrm{D}}\left(\boldsymbol{k}-\boldsymbol{k}_{12}\right) \gamma_{a b c}\left(\boldsymbol{k}_{1}, \boldsymbol{k}_{2}\right) \Psi_{b}\left(\boldsymbol{k}_{1}\right) \Psi_{c}\left(\boldsymbol{k}_{2}\right) \\
=\Xi(\boldsymbol{k} ; \tau) \Psi_{a}(\boldsymbol{k} ; \tau)+\int_{\mathcal{H}} \frac{d^{3} \boldsymbol{k}_{1} d^{3} \boldsymbol{k}_{2}}{(2 \pi)^{3}} \\
\quad \times \delta_{\mathrm{D}}\left(\boldsymbol{k}-\boldsymbol{k}_{12}\right) \gamma_{a b c}\left(\boldsymbol{k}_{1}, \boldsymbol{k}_{2}\right) \Psi_{b}\left(\boldsymbol{k}_{1}\right) \Psi_{c}\left(\boldsymbol{k}_{2}\right)
\end{gathered}
$$

where the first term at the right-hand side represents the contribution from the soft domain, taking the limit, $k \gg q$. The expression for the function $\Xi$ becomes

$$
\Xi(\boldsymbol{k} ; \tau)=\int_{\mathcal{S}} \frac{d^{3} \boldsymbol{q}}{(2 \pi)^{3}}\left(\frac{\boldsymbol{k} \cdot \boldsymbol{q}}{q^{2}}\right) \theta(\boldsymbol{q} ; \tau),
$$

where the subscript ${ }_{\mathcal{S}}$ implies that the integral is restricted to the soft domain.

Similarly, the mode coupling arising from the nonlinear interaction of the scalaron [i.e., the second term in the righthand side of Eq. (7)] can be split into two domains: the soft domain in which one of the modes is much larger than others, and the hard domain in which there is no particularly larger mode than others. We obtain

$$
\begin{aligned}
& \delta_{a 2} \int \frac{d^{3} \boldsymbol{k}_{1} \cdots d^{3} \boldsymbol{k}_{n}}{(2 \pi)^{3(n-1)}} \\
& \quad \times \delta_{\mathrm{D}}\left(\boldsymbol{k}-\boldsymbol{k}_{1 \cdots n}\right) \sigma^{(n)}\left(\boldsymbol{k}_{1}, \ldots, \boldsymbol{k}_{n}\right) \Psi_{1}\left(\boldsymbol{k}_{1}\right) \cdots \Psi_{1}\left(\boldsymbol{k}_{n}\right) \\
& =\omega_{a b}^{(n)}(\boldsymbol{k} ; \tau) \Psi_{b}(\boldsymbol{k} ; \tau) \\
& \quad+\delta_{a 2} \int_{\mathcal{H}} \frac{d^{3} \boldsymbol{k}_{1} \cdots d^{3} \boldsymbol{k}_{n}}{(2 \pi)^{3(n-1)}} \\
& \quad \times \delta_{\mathrm{D}}\left(\boldsymbol{k}-\boldsymbol{k}_{1 \cdots n}\right) \sigma^{(n)}\left(\boldsymbol{k}_{1}, \ldots, \boldsymbol{k}_{n}\right) \Psi_{1}\left(\boldsymbol{k}_{1}\right) \cdots \Psi_{1}\left(\boldsymbol{k}_{n}\right) .
\end{aligned}
$$

Here, the matrix $\omega_{a b}^{(n)}$ includes the contribution from the soft domain, and the nonvanishing contribution appears only in $\omega_{21}^{(n)}$. Let us rewrite it with

$$
\omega_{21}^{(n)}(\boldsymbol{k} ; \tau)=\frac{\kappa^{2}}{2} \frac{\rho_{\mathrm{m}}}{H^{2}} \frac{1}{3} \frac{(k / a)^{2}}{\Pi(k)} \Delta^{(n)}(k ; \tau) .
$$

Here, the function $\Delta^{(n)}$ represents the sum of all possible combinations of the soft/hard domains of the integral. We find that the nonvanishing contribution of $\Delta^{(n)}$ leads to the modification of the effective Newton constant given in 
Eq. (10), as a result of the screening mechanism in modified gravity:

$$
G_{\mathrm{eff}} \rightarrow G\left[1+\frac{1}{3} \frac{(k / a)^{2}}{\Pi(k)}\left\{1+\sum_{n=2} \Delta^{(n)}(k ; \tau)\right\}\right] .
$$

The explicit expression for $\sigma^{(n)}$ is given by

$$
\begin{aligned}
\Delta^{(n)}(k ; \tau)= & -\frac{n}{3 n !} \frac{M_{n}(\tau)}{\Pi(k)}\left(\frac{\kappa^{2} \rho_{\mathrm{m}}}{3}\right)^{n-1} \int_{\mathcal{S}} \frac{d^{3} \boldsymbol{p}_{1} \cdots d^{3} \boldsymbol{p}_{n-1}}{(2 \pi)^{3(n-1)}} \\
& \times K_{f(R)}^{(n)}\left(\boldsymbol{p}_{1}, \ldots, \boldsymbol{p}_{n-1} ; \tau\right) \frac{\delta\left(\boldsymbol{p}_{1} ; \tau\right) \cdots \delta\left(\boldsymbol{p}_{n-1} ; \tau\right)}{\Pi\left(p_{1}\right) \cdots \Pi\left(p_{n-1}\right)}
\end{aligned}
$$

for the $f(R)$ gravity model, and

$$
\begin{aligned}
\Delta^{(n)}(k ; \tau)= & n\left\{-\frac{r_{c}^{2}}{3 \beta(\tau)^{2}} \frac{\kappa^{2} \rho_{\mathrm{m}}}{3}\right\}^{n-1} \int_{\mathcal{S}} \frac{d^{3} \boldsymbol{p}_{1} \cdots d^{3} \boldsymbol{p}_{n-1}}{(2 \pi)^{3(n-1)}} \\
& \times K_{\mathrm{DGP}}^{(n)}\left(\boldsymbol{p}_{1}, \ldots, \boldsymbol{p}_{n-1}\right) \delta\left(\boldsymbol{p}_{1} ; \tau\right) \cdots \delta\left(\boldsymbol{p}_{n-1} ; \tau\right)
\end{aligned}
$$

for the DGP model. Note that the factor $n$ comes from the number of possible combinations of the soft/hard domains of the integral. The functions $K_{f(R)}^{(n)}$ and $K_{\mathrm{DGP}}^{(n)}$ are the dimensionless kernels, whose explicit expressions are presented in Appendix A. In the high- $k$ limit, these contributions are supposed to be subdominant compared to that coming from the standard vertex function [see Eq. (33)], and may be treated perturbatively as a higher-order contribution. This point will be discussed in Sec. III C.

\section{B. Resummed propagator}

Based on the eikonal approximation in Sec. III A, we can now reabsorb the effect of the nonlinear coupling with long-wavelength modes in the linear terms, $\Xi \Psi_{a}$ and $\omega_{a b} \Psi_{b}$. As a result, the evolution equation for perturbation, Eq. (7), can be recast as

$$
\begin{aligned}
& {\left[\delta_{a b}\left\{\frac{\partial}{\partial \tau}-\Xi(k ; \tau)\right\}+\Omega_{a b}(k ; \tau)-\sum_{n=2} \omega_{a b}^{(n)}(k ; \tau)\right] \Psi_{b}(\boldsymbol{k} ; \tau)} \\
& =\int_{\mathcal{H}} \frac{d^{3} \boldsymbol{k}_{1} d^{3} \boldsymbol{k}_{2}}{(2 \pi)^{3}} \delta_{\mathrm{D}}\left(\boldsymbol{k}-\boldsymbol{k}_{12}\right) \gamma_{a b c}\left(\boldsymbol{k}_{1}, \boldsymbol{k}_{2} ; \tau\right) \Psi_{b}\left(\boldsymbol{k}_{1} ; \tau\right) \Psi_{c}\left(\boldsymbol{k}_{2} ; \tau\right) \\
& \quad+\delta_{b 2} \sum_{n=2} \int_{\mathcal{H}} \frac{d^{3} \boldsymbol{k}_{1} \cdots d^{3} \boldsymbol{k}_{n}}{(2 \pi)^{3(n-1)}} \\
& \quad \times \delta_{\mathrm{D}}\left(\boldsymbol{k}-\boldsymbol{k}_{1 \cdots n}\right) \boldsymbol{\sigma}^{(n)}\left(\boldsymbol{k}_{1}, \ldots, \boldsymbol{k}_{n} ; \tau\right) \Psi_{1}\left(\boldsymbol{k}_{1} ; \tau\right) \cdots \Psi_{1}\left(\boldsymbol{k}_{n} ; \tau\right),
\end{aligned}
$$

The solution to this equation can be given in terms of the resummed propagator, $\xi_{a b}$, and it reads

$$
\begin{aligned}
& \Psi_{a}(\boldsymbol{k} ; \tau) \\
& =\xi_{a b}\left(k ; \tau, \tau_{0}\right) \Psi_{b}\left(\boldsymbol{k} ; \tau_{0}\right)+\int_{\tau_{0}}^{\tau} d \tau^{\prime} \xi_{a b}\left(k ; \tau, \tau^{\prime}\right) \\
& \quad \times\left[\int_{\mathcal{H}} \frac{d^{3} \boldsymbol{k}_{1} d^{3} \boldsymbol{k}_{2}}{(2 \pi)^{3}}\right. \\
& \quad \times \delta_{\mathrm{D}}\left(\boldsymbol{k}-\boldsymbol{k}_{12}\right) \gamma_{b c d}\left(\boldsymbol{k}_{1}, \boldsymbol{k}_{2} ; \tau^{\prime}\right) \Psi_{c}\left(\boldsymbol{k}_{1} ; \tau^{\prime}\right) \Psi_{d}\left(\boldsymbol{k}_{2} ; \tau^{\prime}\right) \\
& \quad+\delta_{b 2} \sum_{n=2} \int_{\mathcal{H}} \frac{d^{3} \boldsymbol{k}_{1} \cdots d^{3} \boldsymbol{k}_{n}}{(2 \pi)^{3(n-1)}} \\
& \left.\quad \times \delta_{\mathrm{D}}\left(\boldsymbol{k}-\boldsymbol{k}_{1 \cdots n}\right) \sigma^{(n)}\left(\boldsymbol{k}_{1}, \ldots, \boldsymbol{k}_{n} ; \tau^{\prime}\right) \Psi_{1}\left(\boldsymbol{k}_{1} ; \tau^{\prime}\right) \cdots \Psi_{1}\left(\boldsymbol{k}_{n} ; \tau^{\prime}\right)\right] .
\end{aligned}
$$

Here, the resummed propagator satisfies

$$
\begin{aligned}
& {\left[\delta_{a b}\left\{\frac{\partial}{\partial \tau}-\Xi(k ; \tau)\right\}+\Omega_{a b}(k ; \tau)-\sum_{n=2} \omega_{a b}^{(n)}(k ; \tau)\right]} \\
& \quad \times \xi_{b c}\left(\boldsymbol{k} ; \tau, \tau^{\prime}\right)=0
\end{aligned}
$$

with the boundary condition, $\boldsymbol{\xi}_{a b}(\boldsymbol{k} ; \tau, \tau)=\delta_{a b}$.

In the absence of the term $\omega_{a b}$, the solution of this resummed propagator is expressed in terms of the standard linear propagator of Eq. (7), $g_{a b}$, which satisfies $\left(\partial g_{a c} / \partial \tau\right)+\Omega_{a b} g_{b c}=0$ :

$\xi_{a b}\left(k ; \tau, \tau_{0}\right)=g_{a b}\left(k ; \tau, \tau_{0}\right) \exp \left[\int_{\tau_{0}}^{\tau} d \tau^{\prime} \Xi\left(\boldsymbol{k}, \tau^{\prime}\right)\right]$.

In the presence of the asymmetric matrix $\omega_{a b}$, no tractable analytic expression is obtained, however, assuming that the term $\omega_{a b}$ just gives a subdominant contribution compared to the function $\Xi$, we obtain the approximate expression:

$$
\begin{aligned}
\xi_{a b}\left(k ; \tau, \tau_{0}\right)= & \left\{g_{a b}\left(k ; \tau, \tau_{0}\right)+\int_{\tau_{0}}^{\tau} d \tau^{\prime} g_{a c}\left(k ; \tau, \tau^{\prime}\right)\right. \\
& \left.\times \omega_{c d}\left(k ; \tau^{\prime}\right) g_{d b}\left(k ; \tau^{\prime}, \tau_{0}\right)\right\} \\
& \times \exp \left[\int_{\tau_{0}}^{\tau} d \tau^{\prime \prime} \Xi\left(\boldsymbol{k}, \tau^{\prime \prime}\right)\right] .
\end{aligned}
$$

The resummed propagator given above can be used to systematically compute the multipoint propagators $\Gamma^{(n)}$ defined in Eq. (28), where the nonperturbative high- $k$ behaviors have been already encapsulated (see Sec. IV). In GR, the correction $\omega_{a b}$ vanishes, and all the multipoint propagators are shown to have the exponential damping behaviors [26]. Thus, the nonvanishing contribution of $\omega_{a b}$ is a nontrivial result in modified gravity models. The influence of this on the multipoint propagators will be quantitatively estimated in next subsection. 


\section{Impact of screening effect on resummed propagator}

Let us discuss the impact of screening effect on the resummed propagator, focusing on the new contribution, $\omega_{a b}$. To start with, we define

$$
G_{a b}\left(k ; \tau, \tau_{0}\right)=\left\langle\xi_{a b}\left(k ; \tau, \tau_{0}\right)\right\rangle_{\Xi, \omega_{a b}}
$$

In the cases with the negligible effect of modified gravity at an early time $\tau_{0} \rightarrow-\infty$, contracting $G_{a b}$ with vector $u_{a}=$ $(1,1)$ gives the two-point propagator $\Gamma_{a}^{(1)}$ in the high- $k$ limit, $G_{a b} u_{b} \simeq \Gamma_{a}^{(1)}$. To evaluate the impact of the new correction term, we adopt Eq. (43) and substitute it into the above. We then write

$$
\begin{aligned}
G_{a b}\left(k ; \tau, \tau_{0}\right)= & G_{a b, 0}\left(k ; \tau, \tau_{0}\right)+\delta G_{a b}\left(k ; \tau, \tau_{0}\right) ; \\
G_{a b, 0}(k ; \tau)= & g_{a b}\left(k ; \tau, \tau_{0}\right) \exp \left[-\frac{k^{2}}{2} \int \frac{d q}{6 \pi^{2}}\right. \\
& \left.\times P_{0}(q)\left\{D_{+}(q ; \tau)-D_{+}\left(q ; \tau_{0}\right)\right\}^{2}\right], \\
\delta G_{a b}(k ; \tau)= & \int_{\tau_{0}}^{\tau} d \tau^{\prime} g_{a c}\left(k ; \tau, \tau^{\prime}\right) g_{d b}\left(k ; \tau^{\prime}, \tau_{0}\right) \\
& \times \sum_{n=2}\left\langle\omega_{c d}^{(n)}\left(k ; \tau^{\prime}\right) \exp \left[\int_{\tau_{0}}^{\tau} d \tau^{\prime \prime} \Xi\left(\boldsymbol{k}, \tau^{\prime \prime}\right)\right]\right\rangle_{\Xi, \omega_{a b}} .
\end{aligned}
$$

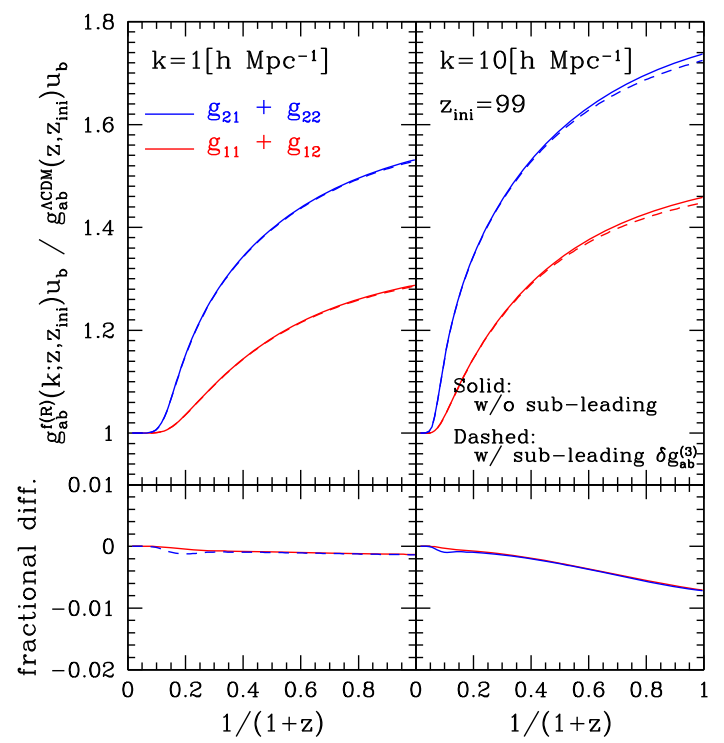

Using Eq. (35), we recast $\delta G_{a b}$ as

$$
\begin{aligned}
& \delta G_{a b}\left(k ; \tau, \tau_{0}\right) \\
& =\int_{\tau_{0}}^{\tau} d \tau^{\prime} g_{a 2}\left(k ; \tau, \tau^{\prime}\right) g_{1 b}\left(k ; \tau^{\prime}, \tau_{0}\right) \frac{\kappa^{2}}{2} \frac{\rho_{\mathrm{m}}\left(\tau^{\prime}\right)}{H^{2}\left(\tau^{\prime}\right)} \frac{1}{3} \frac{(k / a)^{2}}{\Pi\left(k ; \tau^{\prime}\right)} \\
& \quad \times \sum_{n=2}\left\langle\Delta^{(n)} \exp \left[\int_{\tau_{0}}^{\tau} d \tau^{\prime \prime} \Xi\left(\boldsymbol{k}, \tau^{\prime \prime}\right)\right]\right\rangle .
\end{aligned}
$$

Based on the leading-order calculation in which the field $\Psi_{a}=(\delta,-\theta)$ is treated as linear-order quantity, we can explicitly evaluate $\delta G_{a b}$ under the Gaussian initial condition. We then find that the contribution from $n=2$ in Eq. (46) vanishes in both $f(R)$ gravity and DGP models, and the correction from $n=3$ can give the leading-order nonvanishing contribution. Up to this contribution, the propagator $G_{a b}$ can be recast as

$$
\begin{aligned}
& G_{a b}\left(k ; \tau, \tau_{0}\right) \\
& \simeq\left[g_{a b}\left(k ; \tau, \tau_{0}\right)+\delta g_{a b}^{(3)}\left(k ; \tau, \tau_{0}\right)\right] \\
& \quad \times \exp \left[-\frac{k^{2}}{2} \int \frac{d q}{6 \pi^{2}} P_{0}(q)\left\{D_{+}(q ; \tau)-D_{+}\left(q ; \tau_{0}\right)\right\}^{2}\right] .
\end{aligned}
$$

The correction $\delta g_{a b}^{(3)}$ represents the first nonvanishing contributions from $n=3$ of $\delta G_{a b}$. The explicit expression

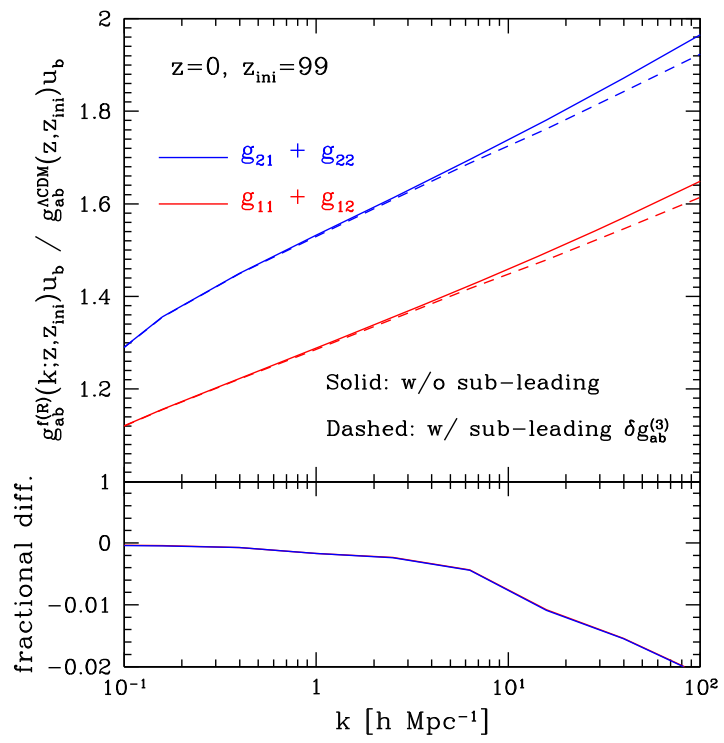

FIG. 1 (color online). Resummed linear propagator in $f(R)$ gravity model. Left panel shows the redshift evolution of the propagator at $k=1 \mathrm{~h} \mathrm{Mpc}^{-1}$ (left) and $10 \mathrm{~h} \mathrm{Mpc}^{-1}$ (right), while right panel plots the scale dependence of the propagator at $z=0$. Top panels plot the ratio of propagator in $f(R)$ gravity to that in GR for density (red) or velocity-divergence fields (blue). Dashed and solid lines respectively represent the result with and without new correction arising from the screening effect, i.e., $\left[g_{a b}+\delta g_{a b}^{(3)}\right]^{\mathrm{f}(\mathrm{R})} u_{b} / g_{a b}^{\Lambda \mathrm{CDM}} u_{b}$ and $g_{a b}^{\mathrm{f}(\mathrm{R})} u_{b} / g_{a b}^{\Lambda \mathrm{CDM}} u_{b}$. On the other hand, to see the size of the correction, bottom panels show the fractional difference between the propagators with and without the correction, i.e., $\left[\delta g_{a b}^{(3)}\right]^{\mathrm{f}(\mathrm{R})} u_{b} / g_{a b}^{\mathrm{f}(\mathrm{R})} u_{b}$. For all panels, we assume $f(R)$ gravity of the functional form in Eq. (23), and the model parameter is set to $\left|f_{R, 0}\right|=10^{-4}$. In computing the propagators, we set the initial redshift to $z_{\text {ini }}=99$, and adopt the cosmological parameters; $\Omega_{\mathrm{m}}=0.24, \Omega_{\Lambda}=0.76, \Omega_{\mathrm{b}}=0.0481, h=0.73, n_{s}=0.961, \sigma_{8}=0.801$ [44]. 
for $\delta g_{a b}^{(3)}$ is presented in Appendix C for the $f(R)$ gravity and DGP models [Eqs. (C7), (C8)].

To see quantitatively the impact of subleading correction, we here consider the $f(R)$ gravity of the functional form in Eq. (23), and compute the correction $\delta g_{a b}^{(3)}$. The results are then compared with the leading-order term, $g_{a b}$. Figure 1 show the ratio of propagator in $f(R)$ gravity to that in GR $(\Lambda \mathrm{CDM})$, without and with the new correction term, i.e., $g_{a b}^{\mathrm{f}(\mathrm{R})} u_{b} / g_{a b}^{\mathrm{GR}} u_{b}$ and $\left[g_{a b}+\delta g_{a b}^{(3)}\right]^{\mathrm{f}(\mathrm{R})} u_{b} / g_{a b}^{\mathrm{GR}} u_{b}$, where the vector $u_{b}$ is defined by $u_{b}=(1,1)$. Note that if the effect of modified gravity is neglected at $\tau_{0}$, the combination $g_{a b} u_{b}$ just gives $g_{a b} u_{b}=\left(D_{+}, d D_{+} / d \tau\right)$, where $D_{+}$is the linear growth factor. Left panel shows the time evolution of the propagator at specific wave numbers $k=1 h \mathrm{Mpc}^{-1}$ (left) and $10 \mathrm{~h} \mathrm{Mpc}^{-1}$ (right), while in right panel, we plot the scale dependence of the propagator at $z=0$. In all cases, the model parameter of $f(R)$ gravity is set to $\left|f_{R, 0}\right|=10^{-4}$. As we see from Fig. 1, the new correction $\delta g_{a b}^{(3)}$ can give a negative contribution, and it basically suppresses the amplitude of the propagators. That is, with the new correction $\omega_{a b}$, the propagator in $f(R)$ tends to approach the one in GR $(\Lambda \mathrm{CDM})$, as depicted in dashed lines, and the effect can become larger at smaller scales. However, the correction itself is very small, and for a currently constrained value of the model parameter, $\left|f_{R, 0}\right| \lesssim 10^{-4}$, we can safely ignore it at least at the scales of our interest.

Note that a negligible contribution of the new correction found above may not be always guaranteed in general modified gravity models. Conservatively, it would be true only for the models with chameleon-type screening mechanisms. Indeed, it is shown analytically and numerically that the effect of screening is quite efficient in DGP model, and the model can recover the standard GR predictions at relatively larger scales (e.g., [67-69]). In this respect, the impact of the high- $k$ correction is nontrivial for models with Vainshtein-type mechanisms, and it may give a nonnegligible contribution to the resummed propagator. We will postpone this issue to a separate paper.

\section{COSMOLOGICAL POWER SPECTRUM FROM REGULARIZED $\Gamma$ EXPANSION}

In this section, based on the resummed propagators, we construct the multipoint propagators that consistently reproduce the expected high- $k$ and low- $k$ behaviors. Using these regularized propagators, we then give the analytic expression of the real-space power spectrum at one-loop order.

Let us first derive the expression of the mutli-point propagators in which the nonperturbative properties in the high- $k$ limit are effectively incorporated. To see the dominant growing-mode contributions, we may set the initial condition, $\Psi_{a}\left(\boldsymbol{k}, \tau_{0}\right)=\delta_{0}(\boldsymbol{k}) u_{a}$ at a very early time, $\tau_{0} \rightarrow-\infty$, and substitute this into the formal solution,
Eq. (40). Then, through the definition (28), the leadingorder expression for the resummed two- and three-point propagators, $\Gamma^{(1)}$ and $\Gamma^{(2)}$, is obtained by taking the functional derivative of the formal solution once and twice, respectively. The resultant expression ignoring the subdominant contribution $\omega_{a b}$ becomes similar to those found in the GR case:

$$
\begin{aligned}
\Gamma_{a}^{(1)}(k ; \tau)= & \left\{g_{a 1}\left(k ; \tau, \tau_{0}\right)+g_{a 2}\left(k ; \tau, \tau_{0}\right)\right\} \\
& \times\left\langle\exp \left[\int_{\tau_{0}}^{\tau} d \tau^{\prime} \Xi\left(\boldsymbol{k}, \tau^{\prime}\right)\right]\right\rangle, \\
\Gamma_{a}^{(2)}\left(\boldsymbol{k}_{1}, \boldsymbol{k}_{2} ; \tau\right)= & \int_{\tau_{0}}^{\tau} d \tau^{\prime} g_{a d}\left(k ; \tau, \tau^{\prime}\right) \\
& \times\left[\left\{\gamma_{d e f}\left(\boldsymbol{k}_{1}, \boldsymbol{k}_{2} ; \tau^{\prime}\right)\right.\right. \\
& \left.+\delta_{d 2} \delta_{e 1} \delta_{f 1} \sigma^{(2)}\left(\boldsymbol{k}_{1}, \boldsymbol{k}_{2} ; \tau^{\prime}\right)\right\} \\
& \left.\times g_{e b}\left(\boldsymbol{k}_{1} ; \tau^{\prime}, \tau_{0}\right) g_{f c}\left(\boldsymbol{k}_{2} ; \tau^{\prime}, \tau_{0}\right)\right]_{\mathcal{H}} u_{b} u_{c} \\
& \times\left\langle\exp \left[\int_{\tau_{0}}^{\tau} d \tau^{\prime} \Xi\left(\boldsymbol{k}, \tau^{\prime}\right)\right]\right\rangle .
\end{aligned}
$$

The ensemble average is taken over the realizations of the field $\Xi(\boldsymbol{k})$. From the explicit expression of $\Xi$ [see Eq. (33)], the ensemble average of the exponential factor becomes

$$
\begin{aligned}
& \left\langle\exp \left[\int_{\tau_{0}}^{\tau} d \tau^{\prime} \Xi\left(\boldsymbol{k}, \tau^{\prime}\right)\right]\right\rangle \\
& \quad=\exp \left[-\frac{k^{2}}{2} \int \frac{d q}{6 \pi^{2}} P_{0}(q)\left\{D_{+}(q ; \tau)-D_{+}\left(q ; \tau_{0}\right)\right\}^{2}\right],
\end{aligned}
$$

where $P_{0}$ and $D_{+}$are the power spectrum of initial density field $\delta_{0}$, and the linear growth factor, respectively. In deriving the expression, we assumed Gaussian initial condition, and used the fact that the linear velocity-divergence field $\theta$ is expressed as $\theta(k ; \tau)=\left\{d D_{+}(k ; \tau) / d \tau\right\} \delta_{0}(k)$.

For the late-time evolution dominated by the growingmode, the integral in front of the exponential factor in Eq. (49) can be reduced to the second-order standard PT kernel, $F_{a}^{(2)}$, sometimes referred to as $\left(F_{2}, G_{2}\right)$ (e.g., $[46,49])$. Thus, taking the limit $\tau_{0} \rightarrow-\infty$, we obtain the simplified expression for $\Gamma^{(n)}$ :

$$
\begin{gathered}
\Gamma_{a}^{(1)}(k ; \tau)=D_{a}(k ; \tau) e^{-k^{2} \sigma_{\mathrm{d}}^{2}(\tau) / 2}, \\
\Gamma_{a}^{(2)}\left(\boldsymbol{k}_{1}, \boldsymbol{k}_{2} ; \tau\right)=F_{a}^{(2)}\left(\boldsymbol{k}_{1}, \boldsymbol{k}_{2} ; \tau\right) e^{-k^{2} \sigma_{\mathrm{d}}^{2}(\tau) / 2},
\end{gathered}
$$

where we define $D_{a} \equiv g_{a 1}+g_{a 2}$, which represents the linear growth factor $D_{+}$and its time derivative $d D_{+} / d \tau$, $D_{a_{2}}=\left(D_{+}, d D_{+} / d \tau\right)$. The explicit calculation of the kernel $F_{a}^{(2)}$ taking account of the effect of modified gravity is described in the Appendix of Ref. [44] [see Eqs. (A17), 
(A18) of their paper]. The quantity $\sigma_{\mathrm{d}}^{2}$ is the dispersion of displacement field defined by

$$
\sigma_{\mathrm{d}}^{2}=\int \frac{d q}{6 \pi^{2}} P_{0}(q)\left\{D_{+}(q ; \tau)\right\}^{2}
$$

With the two- and three-point propagators given above, Eq. (30) truncating at $n=2$ can give the so-called one-loop power spectrum. However, a naive use of Eqs. (51) and (52) may lead to a small flaw in the PT calculation in a sense that one cannot reproduce the standard PT results at low- $k$. To reproduce the standard PT result, the higher-order correction needs to be included consistently in the prediction of propagators. Reference [51] has proposed a novel regularization scheme for propagators that allows us to interpolate the standard PT result and the expected resummed behavior at high- $k$. With this regularized treatment, the power spectrum at one-loop order is expressed as

$$
\begin{aligned}
P_{a b}(k ; \tau)= & \Gamma_{a, \text { reg }}^{(1)}(k ; \tau) \Gamma_{b, \text { reg }}^{(1)}(k ; \tau) P_{0}(k) \\
& +2 \int \frac{d^{3} \boldsymbol{q}}{(2 \pi)^{3}} \Gamma_{a, \text { reg }}^{(2)}(\boldsymbol{q}, \boldsymbol{k}-\boldsymbol{q} ; \tau) \Gamma_{b, \text { reg }}^{(2)}(\boldsymbol{q}, \boldsymbol{k}-\boldsymbol{q} ; \tau) \\
& \times P_{0}(q) P_{0}(|\boldsymbol{k}-\boldsymbol{q}|)
\end{aligned}
$$

with the regularized propagators given by

$$
\begin{aligned}
& \Gamma_{a, \text { reg }}^{(1)}(k ; \tau)= {\left[D_{a}(k ; \tau)\left\{1+\frac{k^{2} \sigma_{\mathrm{d}}^{2}}{2}\right\}+\bar{\Gamma}_{a, 1 \text {-loop }}^{(1)}(k ; \tau)\right] } \\
& \times e^{-k^{2} \sigma_{\mathrm{d}}^{2} / 2} \\
& \Gamma_{a, \text { reg }}^{(2)}(\boldsymbol{q}, \boldsymbol{k}-\boldsymbol{q} ; \tau)=F_{a}^{(2)}(\boldsymbol{q}, \boldsymbol{k}-\boldsymbol{q} ; \tau) e^{-k^{2} \sigma_{\mathrm{d}}^{2} / 2} .
\end{aligned}
$$

Hereafter, we call this regularized PT treatment RegPT.

For the PT calculation at one-loop order, the regularized three-point propagator $\Gamma_{a \text {,reg }}^{(2)}$ is identical to the one given in Eq. (52), and only the two-point propagaor $\Gamma_{a \text {,eg gets some }}^{(1)}$ corrections. The function $\bar{\Gamma}_{a, 1 \text {-loop }}^{(1)}$ represents the one-loop correction to the two-point propagator computed with standard PT. In this paper, to compute it in the $f(R)$ gravity model below, we will use the numerical PT scheme developed by Refs. [70,71]. In the low- $k$ limit $k \sigma_{\mathrm{d}} \ll 1$, the exponential factor in Eq. (55) can be expanded and we recover the standard PT results, i.e., $\Gamma_{a \text {,reg }}^{(1)} \simeq D_{a}+$ $\bar{\Gamma}_{a, 1 \text {-loop }}^{(1)}+\mathcal{O}\left(k^{4} \sigma_{\mathrm{d}}^{4}\right)$. Further, it is known in the GR case that the function $\bar{\Gamma}_{a, 1 \text {-loop }}^{(1)}$ behaves like $\bar{\Gamma}_{a, 1 \text {-loop }}^{(1)} \rightarrow$ $-\left(k^{2} \sigma_{\mathrm{d}}^{2} / 2\right) D_{a}$ in the high- $k$ limit, and thus Eq. (55) reproduces Eq. (51). In general, the latter property does not necessarily hold in modified gravity models. Rather, in the presence of the screening mechanism, it will differ from the one in the GR case. In this respect, the proposition given in Eq. (55) includes a small flaw, and may produce an error in the prediction of propagator. Nevertheless, we will see in the next section that the final impact of this effect is negligible and does not seriously affect the prediction of power spectrum.

\section{COMPARISON WITH $N$-BODY SIMULATIONS}

We are now in a position to compare the PT predictions with $N$-body simulations. We use the simulation data set kindly provided by Baojiu Li. The data set of $N$-body simulations were created by the $N$-body code, ECOSMOG [72], which is a modified version of the mesh-based $N$-body code, RAMSES [73]. With this code, the simulation data were created in both GR and $f(R)$ gravity adopting the functional form in Eq. (23). The cosmological parameters used in the $N$-body simulations are determined by nine-year WMAP results [74], and the initial conditions were generated by MPGRAPHIC [75] at redshift $z_{\text {ini }}=49$, assuming the Gaussianity of initial density field. Hereafter we refer the simulation data to WMAP9. Basic parameters of $N$-body simulations are summarized in Table I. In the analysis presented below, we consider GR and $f(R)$ gravity with $\left|f_{R 0}\right|=10^{-4}$, and use the output data at $z=0$, $0.5,1$, and 2 .

\section{A. Propagator}

Since the power spectrum calculation with $\Gamma$ expansion heavily relies on the prescription for propagators, let us first check their behavior in $N$-body simulations. In our case with the Gaussian initial conditions, the propagators for density field are easily measured in $N$-body simulation without taking the functional derivative. Following Ref. [76], we evaluate the cross correlation between the evolved and initial density fields. Then, dividing it by the linear power spectrum used for the initial condition generator, we obtain the propagator.

Figure 2 plots the measured results of the two-point propagator for the density field in $N$-body simulations, $\Gamma_{1}^{(1)}$ in the cases of GR (left) and $f(R)$ gravity (right). Top panels show the propagators plotted in linear scales, while in the upper part of the bottom panels, the propagators are divided by the linear growth factor, $\Gamma_{1}^{(1)} / D_{+}$, and are plotted as function of wave number squared $k^{2}$ in semi-logarithmic scales. Further, in Fig. 3, to see the relative difference between $f(R)$ gravity and GR, the ratio, $\Gamma_{1, \mathrm{f}(\mathrm{R})}^{(1)} / \Gamma_{1, \mathrm{GR}}^{(1)}$, is

TABLE I. Cosmological parameters used for PT calculations and $N$-body simulations.

\begin{tabular}{lcccccccccc}
\hline \hline Name & $L_{\text {box }}\left[h^{-1} \mathrm{Mpc}\right]$ & \# of particles & $z_{\text {ini }}$ & \# of realizations & $\Omega_{\mathrm{m}}$ & $\Omega_{\Lambda}$ & $\Omega_{\mathrm{b}}$ & $h$ & $n_{s}$ & $\sigma_{8}$ \\
\hline WMAP9 & 1,024 & $1,024^{3}$ & 49 & 1 & 0.281 & 0.719 & 0.0464 & 0.697 & 0.971 & 0.851 \\
\hline \hline
\end{tabular}



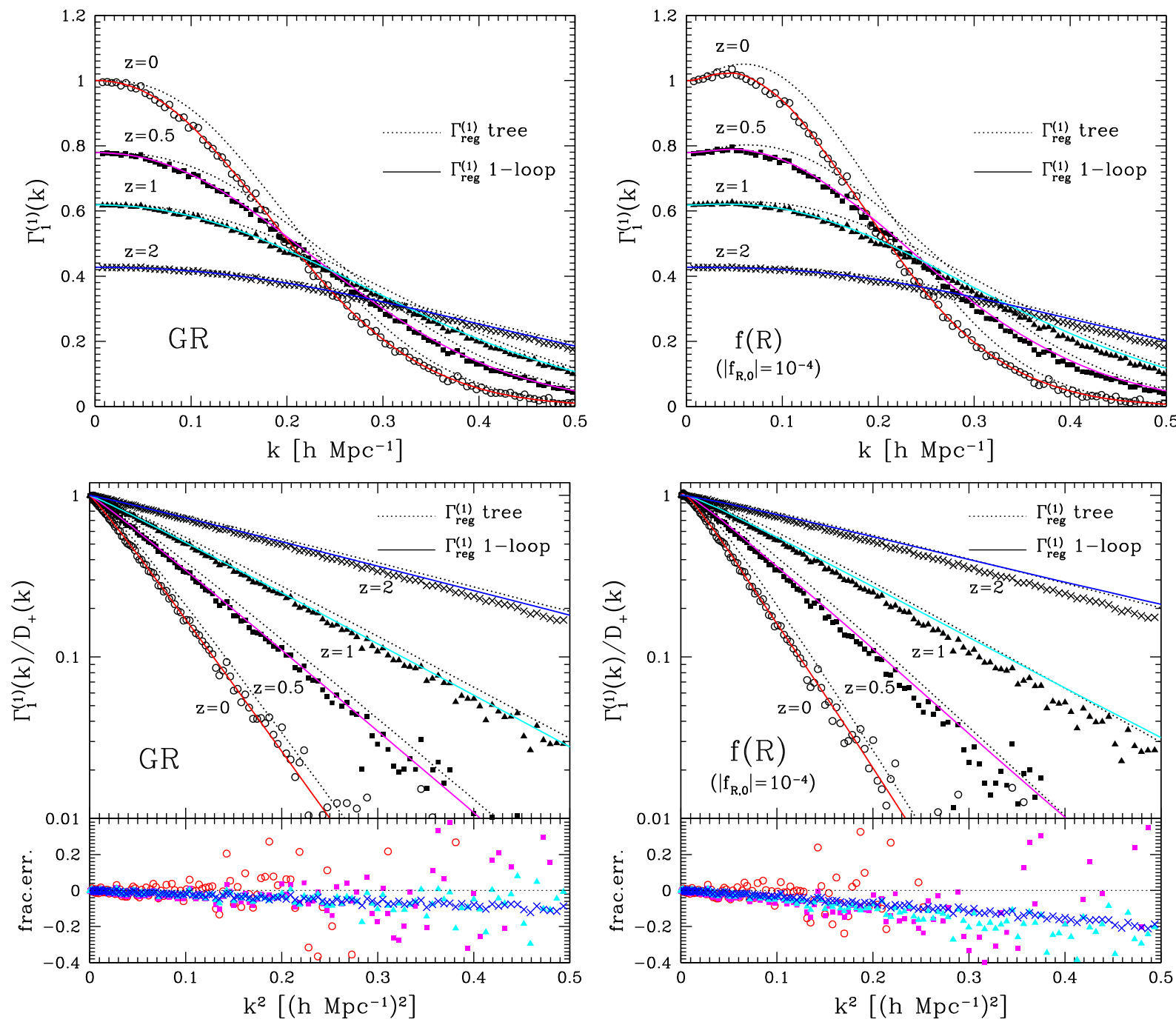

FIG. 2 (color online). Two-point propagator of density field, $\Gamma_{1}^{(1)}(k)$, measured in $N$-body simulations at $z=0$ (open circles), 0.5 (filled squares), 1 (filled triangles), and 2 (crosses). To compare with simulations, regularized propagators at tree level and one-loop order [Eqs. (51) and (55)] are also shown in each panel, depicted as solid and dotted lines, respectively. Left and right panels, respectively, show the results in GR and $f(R)$ gravity with $\left|f_{R, 0}\right|=10^{-4}$. In top panels, the propagators are normally plotted as function of wave number. On the other hand, to clarify the high- $k$ limit behaviors, the normalized propagators $\Gamma^{(1)} / D_{+}$is plotted in upper part of the bottom panel as function of $k^{2}$ in semilog scale. For reference, lower part of the bottom panel shows the fractional difference between simulation results and regularized one-loop predictions, $\Gamma_{\text {sim }}^{(1)}(k) / \Gamma_{\mathrm{PT}}^{(1)}(k)-1$.

plotted as function of $k$. The qualitative behaviors in Fig. 3 are basically deduced from the scale-dependent enhancement of the growth factor in $f(R)$ gravity, which results in both a small increase at low- $k$ and a suppression at high- $k$.

As we see from bottom panels of Fig. 2, the measured propagators exhibit the exponential damping behaviors in both GR and $f(R)$ gravity. The results are then in a good agreement with the theoretical predictions depicted as solid lines, which represent the regularized propagators at oneloop order, $\Gamma_{\text {reg }}^{(1)}$ [Eqs. (55)]. For reference, we also plot the tree-level prediction given in Eq. (51), which degrades the agreement with $\mathrm{N}$-body simulations, as expected from previous studies in GR. The importance of one-loop correction is also seen in Fig. 3, where the predictions reproduce the low- $k$ behavior reasonably well. Note here that we do not indicate the error in $N$-body simulations, since the plotted results are the ratio of measured values, and the cosmic variance cancels out at the leading order. Only with one realization data, we could not properly estimate the higher-order cosmic variance error. Nevertheless, the reasonable agreement with prediction implies that the propagators were reliably estimated in $N$-body simulation, and measured results seem robust against numerical systematics.

A closer look at higher- $k$ behaviors, however, reveals a small discrepancy between predictions and simulations. As quantified in lower part of the bottom panels in Fig. 2, 


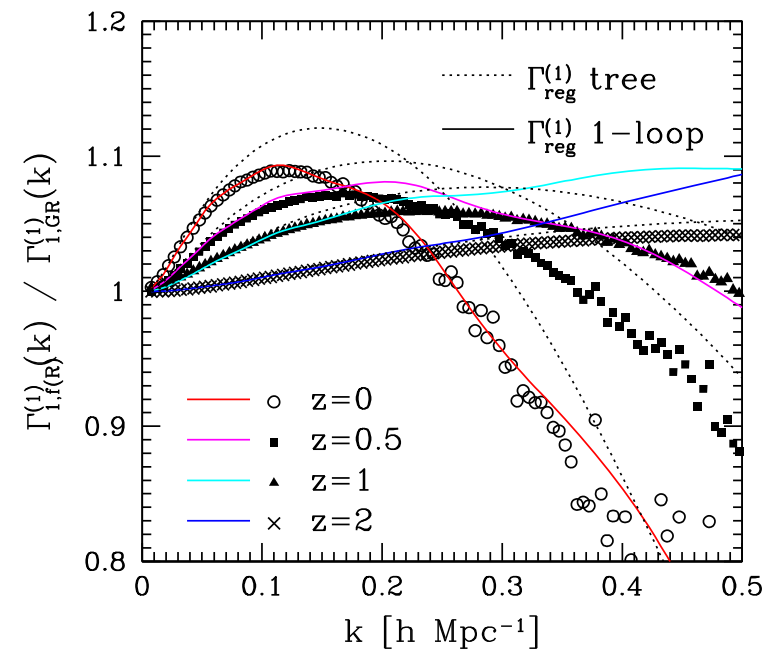

FIG. 3 (color online). Ratio of two-point propagator in $f(R)$ gravity to that in GR, $\Gamma_{1, \mathrm{f}(\mathrm{R})}^{(1)}(k) / \Gamma_{1, \mathrm{GR}}^{(1)}(k)$. Symbols and line types are the same as those in Fig. 2.

the fractional difference between simulation results and regularized one-loop propagators becomes systematically larger at higher- $k$, and in both GR and $f(R)$ cases, the discrepancy is rather manifest at higher redshifts, leading to the $10 \%$ and $20 \%$ errors in GR and $f(R)$. These systematics are also seen in Fig. 3, where the visual impression of the discrepancy is relatively enhanced.

The systematic discrepancy seen in $f(R)$ gravity presumably comes from the small flaw in our regularization treatment, as we discussed in Sec. IV. Nevertheless, since the discrepancy also appears in GR, it might not be solely ascribed to the PT treatment. Rather, one may suspect a small systematic error in the $N$-body simulations. In this

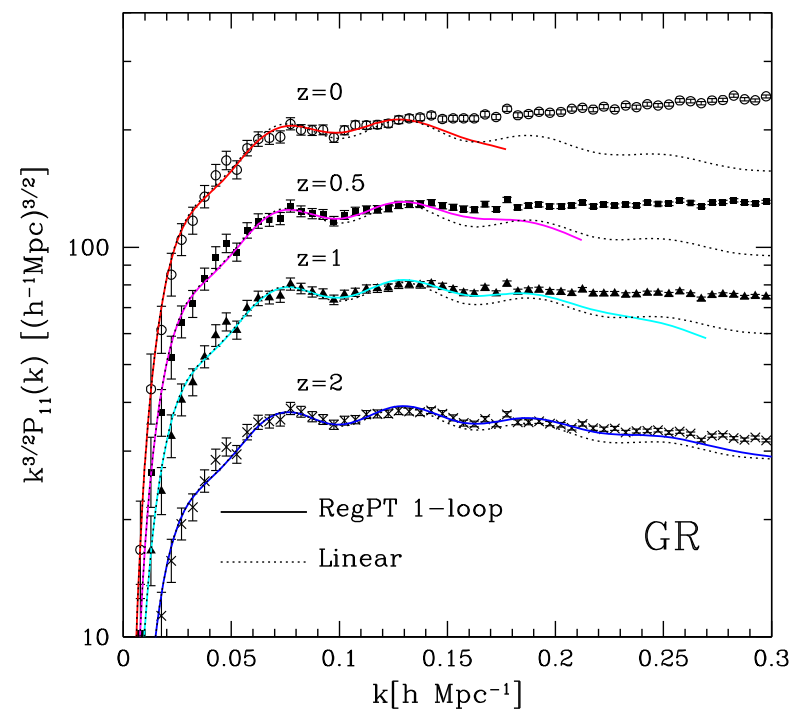

case, a part of the reasons may come from the fact that the initial conditions were generated with the Zel'dovich dynamics, which is known to produce a transient phenomenon due to the nonvanishing decaying mode [77]. As a result, the clustering amplitude becomes slightly reduced compared to the growing-mode dominant initial condition, leading to a suppression of propagators. Another and possibly major reason may be the lack of force resolution. Generally, simulations with insufficient force resolution lead to the incorrect displacements of particles, and thus the cross correlation between the evolved density fields and the linear density field is partly suppressed. This results in a systematic underestimation of propagators, and with the same force resolutions, $\mathrm{N}$-body simulation starting at higher redshift tends to suffer from this systematics. Our previous study reveals that the propagators are more sensitive to the force error at high redshifts than the power spectrum. See Ref. [52] for more detailed discussion. Note also the fact that in $f(R)$ gravity, the nonlinear field equations for scalaron needs to be additionally solved. The error control is thus much more severe in $f(R)$ gravity than GR.

Apart from the small systematics at high- $k$, the RegPT treatment successfully reproduces the overall behavior of the propagator in $N$-body simulations. As long as the propagators are suppressed enough at high- $k$, the discrepancy found in Figs. 2 and 3 would not give a serious impact on the accuracy of the power spectrum and correlation function calculations. We will discuss it in detail by comparing the predictions with $N$-body results below.

\section{B. Power spectrum}

We next present the comparison of power spectra between $N$-body simulations and PT calculations.

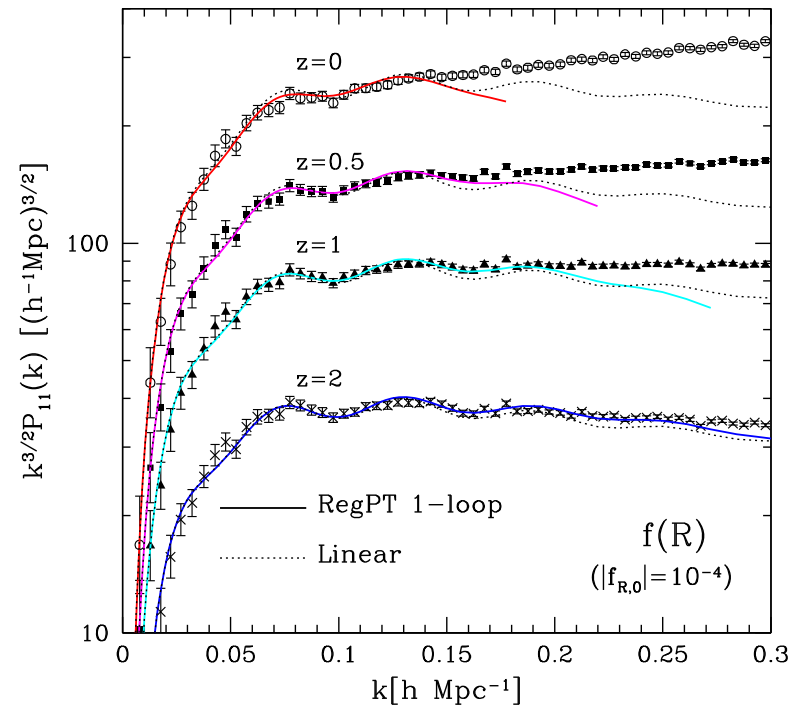

FIG. 4 (color online). Power spectrum of the density field in real space multiplied by $k^{3 / 2}, k^{3 / 2} P_{11}(k)$, at $z=0,0.5$, 1 , and 2 (from top to bottom). Left panel shows the results in GR, while right panel presents the cases in $f(R)$ gravity with $\left|f_{R, 0}\right|=10^{-4}$. Solid and dotted lines are RegPT predictions at one-loop and linear theory predictions, respectively. Note that the error bars indicated in $N$-body results are the dispersion of the power spectrum amplitude over the modes in each Fourier bin. 
Figure 4 presents the power spectra of density field multiplied by $k^{3 / 2}$, i.e., $k^{3 / 2} P_{11}$. The RegPT predictions at one-loop order are depicted as solid lines, and just for reference, we also plot the linear theory predictions in dotted lines.

Because of the scale-dependent linear growth, the resultant amplitude of power spectra in $f(R)$ gravity becomes relatively larger than that in GR, and the differences are manifest at lower redshifts. The RegPT prediction at one-loop order reproduces the $N$-body results fairly well in both cases at the weakly nonlinear scales, where we still clearly see the acoustic signature of power spectrum. Although the RegPT prediction eventually deviates from the $N$-body result at small scales, the range of agreement between $N$-body and PT results is almost the same in both GR and $f(R)$ gravity. With the resummed PT calculation, the nonlinear smearing effect of the BAOs (e.g., $[19,20,78])$, which can be seen in the $N$-body results even at large scales (e.g., $[79,80]$ ), is better described by the PT results, and the prediction shown here is contrasted with the standard PT prediction (see Sec. VII). This point is indeed crucial in accurately predicting the shape and location of the baryon acoustic peak in the correlation function, which we will discuss below.

\section{Correlation function}

The predictions for the correlation function are simply obtained from the power spectrum:

$$
\xi(r)=\int \frac{d k k^{2}}{2 \pi^{2}} P_{11}(k) \frac{\sin (k r)}{k r} .
$$

In the standard PT case, because of the unregularized UV behavior, the above integral cannot be reliably estimated. But now, with the RegPT treatment, we are able to evaluate the correlation function, which can be directly compared with the $N$-body results.

However, only with the single realization data, a reliable estimation of the correlation function is rather difficult in $\mathrm{N}$-body simulations. This is because the measured amplitude of the correlation function is strongly correlated between different scales. Then, due to the cosmic variance error, a small deficit in the initial power spectrum in the $N$-body realization, especially at low- $k$, can coherently affect the shape and amplitude of correlation function over the whole scales, and the measured result of correlation function can drastically differ from what we would expect from the true input power spectrum. The proper way to overcome such a problem is to use a large number of realizations taking ensemble averages over a large number of different realizations. For the problem we are interested in, however, we can still make a meaningful comparison with the single realization data by combining the $N$-body catalogs in GR and $f(R)$ gravity. Let us take the difference:

$$
\Delta \xi(r)=\xi_{f(R)}(r)-\xi_{\mathrm{GR}}(r) .
$$

Since the two catalogs were created with the same random seed, a nonzero value of $\Delta \xi$ implies the systematic difference of the dynamics between GR and $f(R)$ gravity. On the scales we are interested in, the leading-order term in $\Gamma$ expansion is known to play a dominant role for the nonlinear effect on the correlation function (e.g., $[20,25,29])$. Then, from Eq. (54), the PT prediction gives

$$
[\Delta \xi(r)]_{\mathrm{PT}} \simeq\left(\left[\Gamma_{\mathrm{reg}, f(R)}^{(1)}\right]^{2}-\left[\Gamma_{\mathrm{reg}, \mathrm{GR}}^{(1)}\right]^{2}\right) \otimes \xi_{0}(r),
$$

where the symbol $\otimes$ indicates a convolution. The function $\xi_{0}$ represents the correlation function of the input linear density field, which can be computed with the random initial data of $N$-body simulation. Thus, plugging the prediction of the regularized two-point propagators into the above, the predicted value of $[\Delta \xi]_{\mathrm{PT}}$ is directly compared with the measured value.

Figure 5 shows the results of the comparison at $z=0$, 0.5 and 1 (from left to right panels). The measured results of $\Delta \xi$ are plotted as filled circles, while the PT predictions with the regularized one-loop propagator are depicted as solid magenta lines. Note that for clarity, the results at $z=$ 0.5 and 1 are multiplied by the factor 3 and 9 , respectively. We do not plot here the result at $z=2$, since the differences

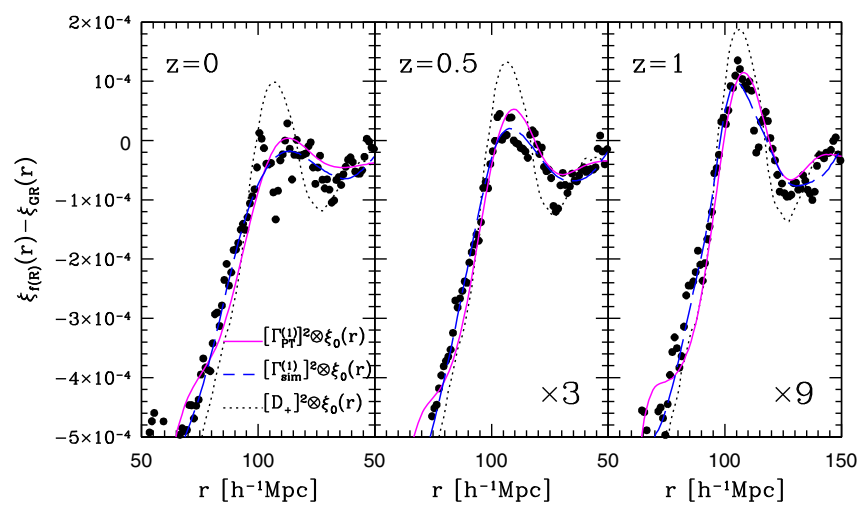

FIG. 5 (color online). Difference of the real-space correlation function between $f(R)$ gravity with $\left|f_{R, 0}\right|=10^{-4}$ and GR, $\Delta \xi(r)=\xi_{f(R)}(r)-\xi_{\mathrm{GR}}(r)$. From left to right panels, the results at $z=0,0.5$, and 1 are shown. In each panel, filled circles represent the results from $N$-body simulations, while the solid, dashed, and dotted lines are estimated from the tree-level expression of the correlation function, $\xi(r) \simeq\left[\Gamma^{(1)}\right]^{2} \otimes \xi_{0}(r)$. Here, $\xi_{0}$ indicates the initial correlation function for which we computed numerically with the random initial data of $N$-body simulation. For the propagator $\Gamma^{(1)}$, the regularized one-loop propagator computed analytically is used in solid lines, while the dashed lines adopt the one directly measured from $\mathrm{N}$-body simulations. Finally, the dotted lines are obtained with the linear theory prediction, just replacing $\Gamma^{(1)}$ with the linear growth factor $D_{+}$. Note that for clarity, the results at $z=0.5$ and 1 are multiplied by the factor 3 and 9 , respectively. 
are quite small. The RegPT prediction fairly traces the measured result of $\Delta \xi$ quite well, and is consistent with the $\mathrm{N}$-body estimates of Eq. (59) depicted as blue dashed lines, in which we directly use the two-point propagator $\Gamma_{\text {reg }}^{(1)}$ measured in $N$-body simulations. For comparison, we also plot the linear theory prediction (dotted), where the twopoint propagators in Eq. (59) are simply replaced with the linear growth factors, $D_{+}$. Clearly, the linear theory prediction fails to reproduce the $N$-body trend. It is known that the baryon acoustic peak tends to be smeared by the nonlinear gravitational growth, and as a result, the location of the acoustic peak is slightly shifted (e.g., $[20,81])$. The behavior of $\Delta \xi$ seen in both PT predictions and $N$-body results basically follows this trend, while a sharp feature in $\Delta \xi$ still remains in linear theory predictions. Thus, the PT prediction with $\Gamma$ expansion can better describe the correlation function, and even at one-loop order it can be used as an accurate theoretical template.

\section{FROM REAL TO REDSHIFT SPACE}

In this section, as an important implication of our resummed PT treatment, we examine the prediction of power spectrum and correlation function in redshift space.

\section{A. Model of RSD}

Since the observed galaxy distribution via spectroscopic measurement basically lies in the redshift space, the effect of redshift-space distortions (RSD) has to be properly incorporated into the theoretical template. Modeling RSD is, however, a nontrivial issue because of the non-Gaussian and nonlinear nature of RSD (e.g., $[31,32,34,82])$. In this paper, we will adopt a specific PT model of RSD recently proposed by Ref. [31]. This model has been shown to explain the redshift-space clustering of dark matter in $N$-body simulations [44,54]. Combining the prescription for galaxy biasing, the model also gives an accurate description of observed power spectra and correlation functions, from which a robust cosmological constraint was obtained [83-87]. Hereafter, we call it the TNS model, and on the basis of the TNS model, we apply our RegPT calculation to the prediction of redshift-space power spectrum and correlation function in modified gravity model.

The TNS model gives a semianalytic prescription for redshift-space power spectrum based on the PT calculations. The functional form of the power spectrum looks very similar to the popular and phenomenological streaming model, but includes higher-order PT corrections. Denoting the directional cosine between observer's lineof-sight and wave vector by $\mu$, we have

$$
\begin{aligned}
P^{(\mathrm{S})}(k, \mu)= & D_{\mathrm{FoG}}\left(k \mu \sigma_{\mathrm{v}}\right) \\
& \times\left\{P_{\text {Kaiser }}(k, \mu)+A(k, \mu)+B(k, \mu)\right\},
\end{aligned}
$$

with the function $P_{\text {Kaiser }}$ called the nonlinear Kaiser term:

$$
P_{\text {Kaiser }}(k, \mu)=P_{11}(k)+2 \mu^{2} P_{12}(k)+\mu^{4} P_{22}(k) .
$$

Here, the function $D_{\mathrm{FoG}}$ characterizes the nonperturbative damping effect caused by both the coherent and small-scale virialized motions, and we here assume the Gaussian form: $D_{\mathrm{FoG}}=\exp \left[-\left(k \mu \sigma_{\mathrm{v}}\right)^{2}\right]$. The parameter $\sigma_{\mathrm{v}}$ is a scaleindependent constant, and is determined by fitting the prediction to simulation or observation. In this respect, Eq. (60) may be regarded as a semiempirical model, however, a salient feature of the TNS model is the presence of the correction terms $A$ and $B$ that account for the nonlinear modulation of BAO in redshift space quite well. These two corrections represent the mode-coupling between density and velocity fields originating from the nonlinear mapping from real to redshift spaces. They are expressed as

$$
\begin{aligned}
A(k, \mu)= & \sum_{n=1}^{3} \sum_{a, b=1}^{2} \mu^{2 n} \frac{k^{3}}{(2 \pi)^{2}} \int_{0}^{\infty} d r \int_{-1}^{1} d x \\
& \times\left\{A_{a b}^{n}(r, x) B_{2 a b}(\boldsymbol{p}, \boldsymbol{k}-\boldsymbol{p},-\boldsymbol{k})\right. \\
& \left.+\tilde{A}_{a b}^{n}(r, x) B_{2 a b}(\boldsymbol{k}-\boldsymbol{p}, \boldsymbol{p},-\boldsymbol{k})\right\}, \\
B(k, \mu)= & \sum_{n=1}^{4} \sum_{a, b=1}^{2} \mu^{2 n} \frac{k^{3}}{(2 \pi)^{2}} \times \int d r_{0}^{\infty} \int_{-1}^{1} d x B_{a b}^{n}(r, x) \\
& \times \frac{P_{a 2}\left(k \sqrt{1+r^{2}-2 r x}\right) P_{b 2}(r x)}{\left(1+r^{2}-2 r x\right)^{a}},
\end{aligned}
$$

where $r$ and $x$ are the dimensionless variables associated with the wave vector, $\boldsymbol{p}$, defined by $r=p / k$ and $x=(\boldsymbol{k} \cdot \boldsymbol{p}) /(k p)$, respectively. The function $B_{a b c}$ is the bispectrum defined by

$$
\begin{aligned}
& \left\langle\Psi_{a}\left(\boldsymbol{k}_{1}\right) \Psi_{b}\left(\boldsymbol{k}_{2}\right) \Psi_{c}\left(\boldsymbol{k}_{3}\right)\right\rangle \\
& \quad=(2 \pi)^{3} \delta_{\mathrm{D}}\left(\boldsymbol{k}_{1}+\boldsymbol{k}_{2}+\boldsymbol{k}_{3}\right) B_{a b c}\left(\boldsymbol{k}_{1}, \boldsymbol{k}_{2}, \boldsymbol{k}_{3}\right) .
\end{aligned}
$$

The nonvanishing coefficients, $A_{a b}^{n}, \tilde{A}_{a b}^{n}$, and $B_{a b}^{n}$ are the same as those presented in Appendix A of Ref. [31], and we use them to compute Eqs. (62) and (63). For the prediction at one-loop order, while we apply the regularized one-loop expression in Eq. (54) to the Kaiser term $P_{\text {Kaiser }}$, the correction terms $A$ and $B$ appear as a next-to-leading order correction, and for a consistent treatment, the tree-level calculation is sufficient for these two terms. Thus, the power spectrum and bispectrum in the $A$ and $B$ terms may be evaluated as

$$
\begin{gathered}
P_{a b, \text { tree }}(k)=\Gamma_{a}^{(1)}(k) \Gamma_{b}^{(1)}(k) P_{0}(k), \\
B_{a b c \text {,tree }}\left(\boldsymbol{k}_{1}, \boldsymbol{k}_{2}, \boldsymbol{k}_{3}\right)= \\
2 \Gamma_{a}^{(2)}\left(\boldsymbol{k}_{2}, \boldsymbol{k}_{3}\right) \Gamma_{b}^{(1)}\left(k_{2}\right) \Gamma_{c}^{(1)}\left(k_{3}\right) \\
\times P_{0}\left(k_{2}\right) P_{0}\left(k_{3}\right)+(\text { cyc.perm }),
\end{gathered}
$$



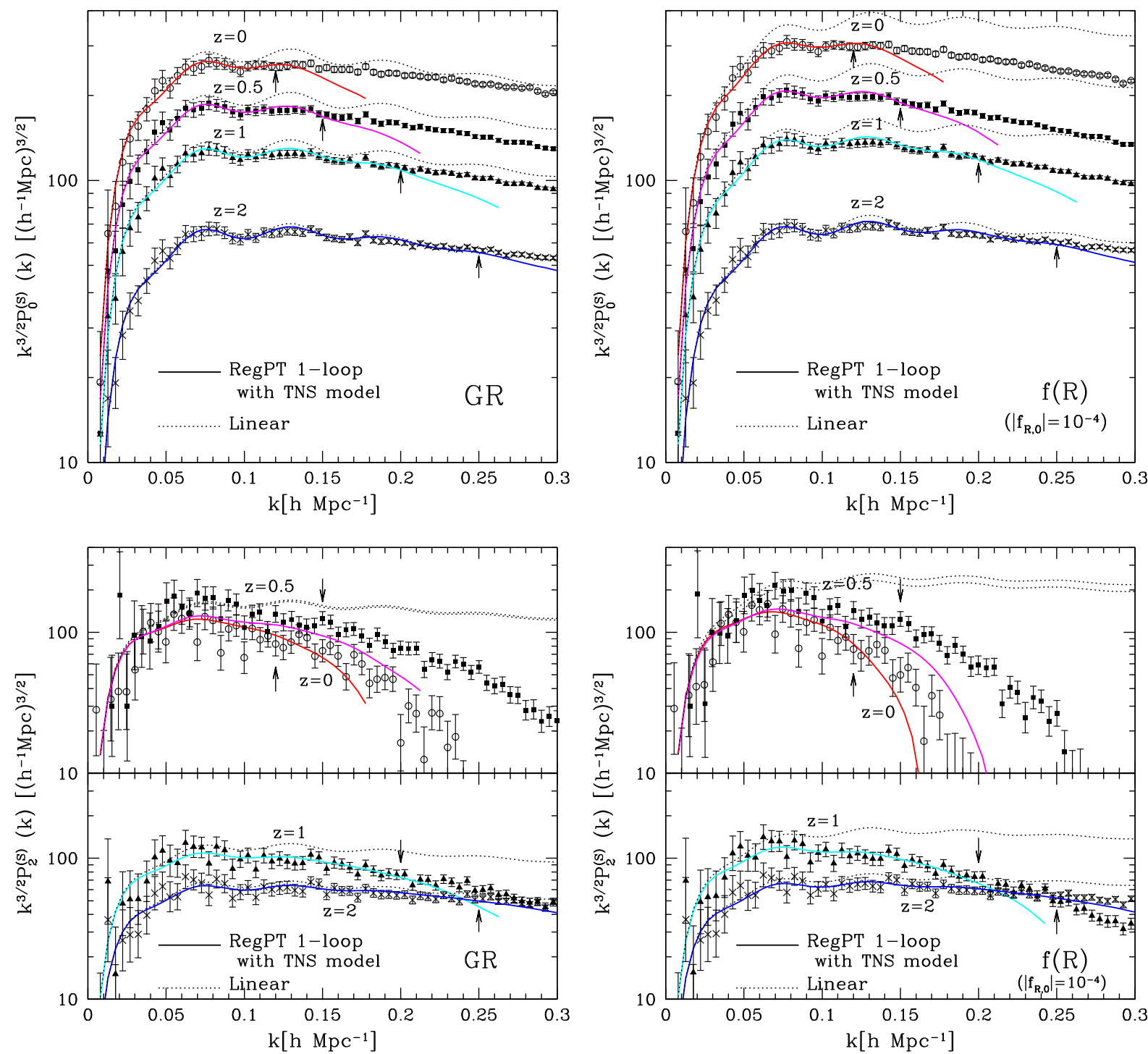

FIG. 6 (color online). Monopole $(\ell=0$, top) and quadrupole $(\ell=2$, bottom) moments of redshift-space power spectrum at $z=0$, $0.5,1$, and 2. Left panel shows the results in GR, while right panel presents the cases in $f(R)$ gravity with $\left|f_{R, 0}\right|=10^{-4}$. In each panel, dotted lines represent the linear theory calculations based on the Kaiser formula, while the solid lines are the RegPT one-loop results based on the TNS model of RSD.

with the propagators $\Gamma_{a}^{(1)}$ and $\Gamma_{a}^{(2)}$ being evaluated with the tree-level expressions [see Eqs. (51) and (52)].

\section{B. Results}

Figure 6 plots the monopole $(\ell=0$, top) and quadrupole $(\ell=2$, bottom) moments of redshift-space power spectrum, multiplied by $k^{3 / 2}$. The multipole moment of power spectrum is defined by

$$
P_{\ell}^{(\mathrm{S})}(k)=\frac{2 \ell+1}{2} \int_{-1}^{1} d \mu P^{(\mathrm{S})}(k, \mu) \mathcal{P}_{\ell}(\mu)
$$

The PT predictions based on the TNS model are shown in solid lines, and just for reference, linear theory results are also shown in dotted lines. In plotting the PT results, the free parameter $\sigma_{\mathrm{v}}$ is first determined by fitting the model predictions to the $N$-body results of monopole and quadrupole spectra up to the maximum wave number, $k_{\max }$. In

TABLE II. Fitting results of the free parameter $\sigma_{\mathrm{v}}$ for the model of redshift-space power spectra shown in Fig. 6.

\begin{tabular}{ccc}
\hline \hline & $\mathrm{GR}$ & $f(R)$ \\
$z$ & $\sigma_{\mathrm{v}}\left[h^{-1} \mathrm{Mpc}\right]$ & $\sigma_{\mathrm{v}}\left[h^{-1} \mathrm{Mpc}\right]$ \\
\hline 0 & 5.18 & 6.65 \\
0.5 & 4.76 & 6.03 \\
1 & 3.71 & 4.62 \\
2 & 2.25 & 2.64 \\
\hline \hline
\end{tabular}



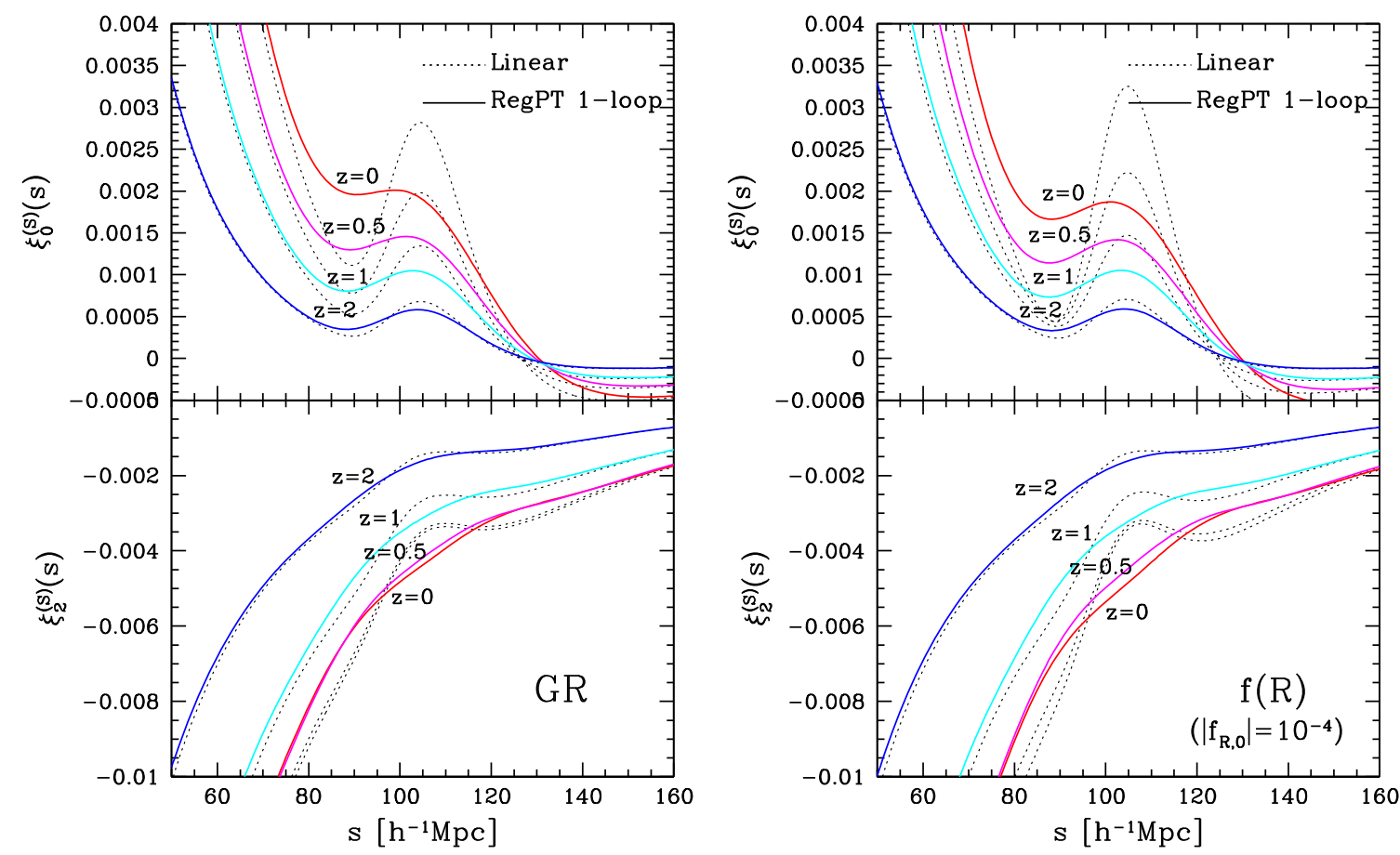

FIG. 7 (color online). Prediction of redshift-space correlation functions at $z=0,0.5,1$, and 2 in GR (left) and $f(R)$ gravity with $\left|f_{R, 0}\right|=10^{-4}$ (right). Based on the TNS model, the monopole $(\ell=0)$ and quadrupole $(\ell=2)$ moments of the correlation function are shown in top and bottom panels, respectively. Note that in computing the correlation function, we used the fitting parameter $\sigma_{\mathrm{v}}$ determined from power spectrum (Fig. 6). In each panel, solid and dotted lines are the RegPT and linear theory predictions.

each panel, $k_{\max }$ is indicated as vertical arrow, below which a percent-level agreement between the PT predictions and $\mathrm{N}$-body simulation is achieved in real-space power spectrum (see Fig. 4).

Overall, similarly to the real space, the PT results show a very good performance in both monopole and quadrupole spectra. This is true in both GR and $f(R)$ gravity model. A closer look at low- $z$ results of the quadrupole reveals a small discrepancy with $N$-body results at $k \sim k_{\max }$, although the measured power spectra are a bit noisy and the error bars are large. This partly comes from a flaw in the PT model of RSD, but with the other choice of the damping function $D_{\text {Fog }}$ (Lorentzian form, for instance), the prediction would be improved [54]. Rather, one remarkable point of the low $z$ results may be that the damping of the power spectrum amplitude relative to the linear theory prediction is rather significant in $f(R)$ gravity compared to that found in GR gravity. Table II summarizes the fitted results of parameter $\sigma_{\mathrm{v}}$, clearly showing a stronger suppression of the power spectrum in $f(R)$ gravity. As it has been already studied (e.g., $[44,88])$, this is partly explained by the linear theory. In $f(R)$ gravity, the structure formation is enhanced at small scales due to the presence of the scale-dependent linear growth, and it produces a larger peculiar velocity. Although the screening mechanism should terminate the enhanced structure growth at some nonlinear scales, it is still ineffective at the scales of our interest.
Finally, Fig. 7 shows the prediction of redshift-space correlation function. Based on the results in Fig. 6, monopole and quadrupole moments of the correlation functions are computed and are shown in solid lines:

$$
\xi_{\ell}^{(\mathrm{S})}(s)=i^{\ell} \int \frac{d k k^{2}}{2 \pi^{2}} P_{\ell}^{(\mathrm{S})}(k) j_{\ell}(k s)
$$

We do not present here the $N$-body results, because only with the single realization data, we do not reliably estimate the correlation function. While a detailed comparison of the results between PT calculation and simulation will be made elsewhere, the predictions shown in Fig. 7 look reasonable, and one expects that the PT results quantitatively describe the impact of both the nonlinear gravity and RSD around baryon acoustic peak. It suggests that in $f(R)$ gravity, the acoustic peak is significantly smeared out, and the peak amplitude is largely suppressed relative to the linear theory prediction. The resultant shape and amplitude look rather similar to those in GR. Hence, taking full account of these nonlinear effects would be essential in constraining the modified gravity from the observed correlation functions.

\section{DISCUSSION: COMPARISON WITH STANDARD PERTURBATION THEORY}

While the standard PT treatment fails to compute the correlation function, at sufficiently large scales and high 

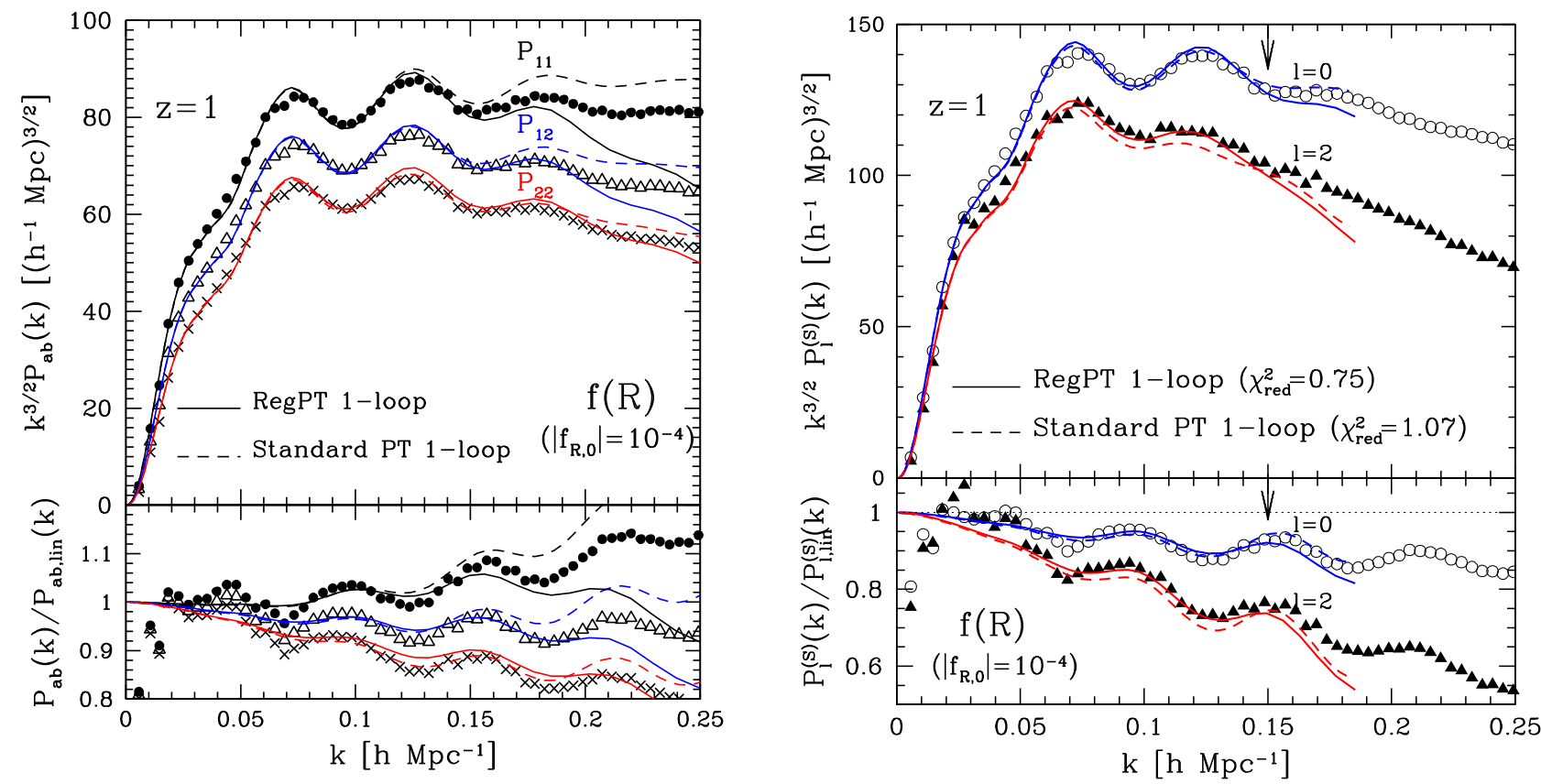

FIG. 8 (color online). Comparison between RegPT and standard PT results at one-loop order in real and redshift spaces. Left panel plots the auto- and cross-power spectra of density and velocity fields in real space at $z=1$. The results in $f(R)$ gravity with $\left|f_{R, 0}\right|=$ $10^{-4}$ are shown. Solid and dotted lines are the PT predictions computed with RegPT and standard PT, respectively. In the right panel, redshift-space power spectra at $z=1$ are plotted. Based on the TNS model, the monopole $(\ell=0)$ and quadrupole $(\ell=2)$ power spectra are computed, and the results are compared with $N$-body simulations. The solid and dashed lines, respectively, represent the PT predictions computed with RegPT and standard PT.

redshifts, it still provides a reasonably accurate prediction of the power spectrum. Indeed, in Ref. [44], we used the standard PT to compute the real- and redshift-space power spectra in $f(R)$ gravity, and a good agreement with $N$-body simulation was found at $z=1$. It is thus interesting to see how much the standard PT results are different from our RegPT treatment.

To compare with the standard PT results, we adopt the same cosmological parameters as used in Ref. [44], and the power spectra at $z=1$ are computed with the RegPT treatment in both real and redshift spaces. The results are then shown in Fig. 8, where the $N$-body results and the standard PT predictions taken from Ref. [44] are also plotted in symbols and dashed lines, respectively. Top panels show the power spectra multiplied by $k^{3 / 2}$, while bottom panels plot the ratio of the power spectrum to the linear theory prediction. Note that in both PT predictions, the TNS model has been used to compute the monopole and quadrupole power spectra in right panel.

Overall, both of the PT results reproduce the $N$-body trend reasonably well at $k \lesssim 0.15 h \mathrm{Mpc}^{-1}$, but a closer look at the real-space power spectrum reveals that the RegPT gives a moderate nonlinear enhancement or suppression of the power spectrum amplitude, and it seems to better describe the smearing effect on BAO. This trend is also seen in redshift-space power spectrum. Indeed, the $N$-body results of the quadrupole power spectrum is better described by the RegPT prediction.
To see this quantitatively, we evaluate the $\chi$-squared statistics:

$$
\chi_{\text {red }}^{2}=\frac{1}{\nu} \sum_{\ell=0,2} \sum_{i} \frac{\left[P_{\ell, \mathrm{N}-\text { body }}^{(\mathrm{S})}\left(k_{i}\right)-P_{\ell, \mathrm{PT}}^{(\mathrm{S})}\left(k_{i}\right)\right]^{2}}{\left[\Delta P_{\ell}^{(\mathrm{S})}\left(k_{i}\right)\right]^{2}},
$$

with the quantity $\nu$ being the number of degrees of freedom. For simplicity, we here ignore the cross covariance between the monopole and quadrupole spectra, which is shown to be fairly small. The statistical error $\Delta P_{\ell}^{(\mathrm{S})}$ is estimated from the cosmic variance error assuming the hypothetical survey with volume $10 h^{-3} \mathrm{Gpc}^{3}$ (see Appendix B of Ref. [31] for the explicit expressions). With the number of Fourier bins below the maximum wave number indicated by vertical arrows, the resultant numerical values of $\chi_{\text {red }}^{2}$ are shown in the panel, clearly showing that the RegPT prediction gives a good performance, and reproduce the $N$-body results quite well. Note that the $\chi_{\text {red }}^{2}$ value smaller than unity does not implies that the model overfits the simulations because the statistical error $\Delta P_{\ell}^{(S)}$ adopted here does not reflect the actual error in $N$-body simulations. Although the differences of the PT prediction between standard PT and RegPT is small at one-loop order, a better description of acoustic feature in power spectrum is rather crucial in simultaneous measurement of geometric distances, and the RegPT procedure provides a more accurate theoretical template from which 
one should be able to obtain unbiased parameter estimations.

\section{CONCLUSION}

Future precision observations of large-scale structure enables us to not only give a useful cosmological constraint on dark energy but also offer a new possibility to probe the theory of gravity itself that describes both the dynamics of cosmic expansion and the growth of large-scale structure. Toward an unbiased cosmological constraint, it is therefore crucial to develop accurate theoretical templates of largescale structure observations. In this paper, beyond a consistency test of gravity from a precision measurement of BAO and RSD, we presented an improved PT prescription of the power spectrum and correlation function in modified gravity models.

Based on the resummed PT scheme with $\Gamma$ expansion, we first construct a resummed propagator that partly includes the nonperturbative effect in the high- $k$ limit. While the resultant propagator in the high- $k$ limit contains corrections arising from the screening mechanism, we explicitly show that in the case of $f(R)$ gravity with a currently constrained model parameter, the impact of this term is fairly small. Thus, in $f(R)$ gravity, the regularized propagators, that reproduce both the resummed high- $k$ behavior and the low- $k$ results computed with standard PT, are successfully constructed in a similar manner to the GR case, taking account of the nonlinear modification of gravity valid at large scales. Then, the analytically calculated propagators have been compared with the measured results in $N$-body simulations. At one-loop order, the analytic predictions reproduces the measured propagators quite well, and a good agreement with $\mathrm{N}$ body simulations was also obtained for the analytic power spectrum and correlation function expressed in terms of the regularized propagators. Furthermore, employing a specific mode of RSD, the improved PT prescription has been shown to successfully describe the redshift-space observables. For the power spectrum at one-loop order, while a performance of our improved PT treatment apparently looks very similar to that of the standard PT prediction, nonlinear modulation of BAO seen in $N$-body simulations is better described by our improved PT predictions, showing that the resummed PT developed here is better suited for an unbiased cosmological constraints from BAO measurements.

Finally, while the explicit PT calculations presented here have focused on a specific modified gravity model, the resummed PT prescription developed in this paper, as well as the framework to treat PT calculations, are quite general, and can be applied to a wide class of modified gravity models. This is at least true for models with chameleon-type screening mechanisms within the current observational bounds. Rather, with the improved PT prescription presented here, we will be able to put a more stringent constraint on modified gravity from a precision measurement of BAO and RSD. For instance, the $f(R)$ gravity of the functional form in Eq. (22) or (23) is currently constrained to $\left|f_{R, 0}\right| \lesssim 10^{-4}$, from the RSD measurement with SDSS DR7 luminous red galaxy sample [62]. While this is roughly comparable to the one obtained from cluster abundance [89], there are now much larger galaxy samples such as BOSS DR11 CMASS sample, from which we will get a much more severe constraint on $f(R)$ gravity. Note also that the template used in Ref. [62] is based on the fitting formula, which does not properly account for the nonlinear modulation of BAO coming from both the gravity and RSD. Hence, with the new PT template and the model of RSD presented here, a robust and unbiased cosmological analysis will be made possible. In doing this, however, a careful study of the galaxy biasing is needed. Indeed, the halo clustering properties in $f(R)$ gravity has been found to systematically differ from those in GR (e.g., [90]). Since even in the GR, halo/galaxy clustering not only has a scale-dependent property but also possibly exhibits a biasing on the velocity field (e.g., [91,92]), a proper account of these biasing effects is rather crucial toward an unbiased test of gravity.

\section{ACKNOWLEDGMENTS}

We are grateful to Baojiu Li for kindly providing us the $N$-body simulation data. This work is supported in part by a Grant-in-Aid for Scientific Research from the Japan Society for the Promotion of Science (No. 23740186 for T. H. and No. 24540257 for A. T.), in part by Grant No. ANR-12-BS05-0002 of the French Agence Nationale de la Recherche. T. N. is supported by JSPS Postdoctoral Fellowships for Research Abroad. K. K. is supported by the UK Science and Technology Facilities Council (STFC) Grants No. ST/K00090/1 and No. ST/L005573/1. T. H. acknowledges a support from MEXT HPCI Strategic Program. The numerical calculations in this work were partly carried out on Cray XC30 at Center for Computational Astrophysics, CfCA, of National Astronomical Observatory of Japan.

\section{APPENDIX A: EXPLICIT EXPRESSION FOR THE KERNELS, $K_{f(R)}^{(n)}$ AND $K_{\text {DGP }}^{(n)}$}

In this appendix, based on the expressions for the vertex functions in Eqs. (13)-(15), we present the explicit expressions for the kernels in the high- $k$ limit, $K_{f(R)}$ and $K_{\text {DGP }}$, in Sec. III A.

\section{1. $\boldsymbol{f}(\boldsymbol{R})$ gravity}

Recalling the fact that the coupling functions $M_{i}$ in $f(R)$ gravity are all scale-independent, and just given as function of time [see Eq. (20)], the kernel functions defined in Eq. (37) become 


$$
\begin{aligned}
& K_{f(R)}^{(2)}=1, \\
& K_{f(R)}^{(3)}=1-\frac{M_{2}^{2}}{3 M_{3}} \frac{1}{\Pi\left(p_{12}\right)}, \\
& K_{f(R)}^{(4)}=1-\frac{M_{2} M_{3}}{6 M_{4}} \frac{1}{\Pi\left(p_{123}\right)}+\left\{\frac{M_{2}^{3}}{18 M_{4}} \frac{1}{\Pi\left(p_{123}\right)}-\frac{M_{2} M_{3}}{3 M_{4}}\right\} \\
& \times\left\{\frac{1}{\Pi\left(p_{12}\right)}+\frac{1}{\Pi\left(p_{23}\right)}+\frac{1}{\Pi\left(p_{31}\right)}\right\} \text {. } \\
& K_{\mathrm{DGP}}^{(2)}\left(\boldsymbol{p}_{1}\right)=1-\mu_{1}^{2}, \\
& K_{\mathrm{DGP}}^{(3)}\left(\boldsymbol{p}_{1}, \boldsymbol{p}_{2}\right)=\frac{2}{3}\left\{2\left(1-\mu_{1}^{2}\right)\left(1-\mu_{2}^{2}\right)\right. \\
& \left.+\left(1-\mu_{12}^{2}\right)\left(1-\mu_{1,2}^{2}\right)\right\} \text {, } \\
& K_{\mathrm{DGP}}^{(4)}\left(\boldsymbol{p}_{1}, \boldsymbol{p}_{2}, \boldsymbol{p}_{3}\right)=\frac{1}{3}\left\{\left(1-\mu_{23}^{2}\right)\left(1-\mu_{1}^{2}\right)\left(1-\mu_{2,3}^{2}\right)+\left(1-\mu_{31}^{2}\right)\left(1-\mu_{2}^{2}\right)\left(1-\mu_{3,1}^{2}\right)+\left(1-\mu_{12}^{2}\right)\left(1-\mu_{3}^{2}\right)\left(1-\mu_{1,2}^{2}\right)\right\} \\
& +\frac{1}{6}\left(1-\mu_{3}^{2}\right)\left\{2\left(1-\mu_{1}^{2}\right)\left(1-\mu_{2}^{2}\right)+\left(1-\mu_{12}^{2}\right)\left(1-\mu_{1,2}^{2}\right)\right\} \\
& +\frac{1}{6}\left(1-\mu_{1}^{2}\right)\left\{2\left(1-\mu_{2}^{2}\right)\left(1-\mu_{3}^{2}\right)+\left(1-\mu_{23}^{2}\right)\left(1-\mu_{2,3}^{2}\right)\right\} \\
& +\frac{1}{6}\left(1-\mu_{2}^{2}\right)\left\{2\left(1-\mu_{3}^{2}\right)\left(1-\mu_{1}^{2}\right)+\left(1-\mu_{31}^{2}\right)\left(1-\mu_{3,1}^{2}\right)\right\} \\
& +\frac{1}{6}\left(1-\mu_{123}^{2}\right)\left\{\left(1-\mu_{12,3}^{2}\right)\left(1-\mu_{12}^{2}\right)+\left(1-\mu_{23,1}^{2}\right)\left(1-\mu_{2,3}^{2}\right)+\left(1-\mu_{31,2}^{2}\right)\left(1-\mu_{3,1}^{2}\right)\right\},
\end{aligned}
$$

where we define $\mu_{i}=\left(\boldsymbol{k} \cdot \boldsymbol{p}_{i}\right) /\left(k p_{i}\right), \mu_{i j}=\left(\boldsymbol{k} \cdot \boldsymbol{p}_{i j}\right) /\left(k p_{i j}\right)$, and $\mu_{i, j}=\left(\boldsymbol{p}_{i} \cdot \boldsymbol{p}_{j}\right) /\left(p_{i} p_{j}\right)$.

\section{APPENDIX B: VERTEX FUNCTION $\sigma^{(n)}$}

In this appendix, we derive the expression for the vertex function $\sigma^{(n)}$. To do this, we first write down the expression for scalaron, $\varphi$, and express it in terms of $\delta$. From Eqs. (2) and (3), we have

$$
\begin{aligned}
\varphi(\boldsymbol{k})= & \frac{\kappa^{2} \rho_{\mathrm{m}}}{3} \frac{\delta(\boldsymbol{k})}{\Pi(k)}-\frac{1}{3 \Pi(k)}\left[\frac{1}{2} M_{2}\left(\boldsymbol{k}_{1}, \boldsymbol{k}_{2}\right) \varphi\left(\boldsymbol{k}_{1}\right) \varphi\left(\boldsymbol{k}_{2}\right)+\frac{1}{6} M_{3}\left(\boldsymbol{k}_{1}, \boldsymbol{k}_{2}, \boldsymbol{k}_{3}\right) \varphi\left(\boldsymbol{k}_{1}\right) \varphi\left(\boldsymbol{k}_{2}\right) \varphi\left(\boldsymbol{k}_{3}\right)\right. \\
& \left.+\frac{1}{24} M_{4}\left(\boldsymbol{k}_{1}, \boldsymbol{k}_{2}, \boldsymbol{k}_{3}, \boldsymbol{k}_{4}\right) \varphi\left(\boldsymbol{k}_{1}\right) \varphi\left(\boldsymbol{k}_{2}\right) \varphi\left(\boldsymbol{k}_{3}\right) \varphi\left(\boldsymbol{k}_{4}\right)+\cdots\right]
\end{aligned}
$$

with the function $\Pi$ defined in Eq. (9). Here, we introduced the short-cut notation that the repeated Fourier arguments, $\boldsymbol{k}_{1}, \ldots, \boldsymbol{k}_{n}$, are, after multiplying by the Dirac $\delta$ function $\delta_{D}\left(\boldsymbol{k}-\boldsymbol{k}_{1 \ldots n}\right)$, integrated over. To express $\varphi$ in terms of $\delta$, we perturbatively expand $\varphi$ :

$$
\varphi=\varphi_{1}+\varphi_{2}+\varphi_{3}+\varphi_{4}+\ldots
$$

Then, we have

$$
\begin{aligned}
\varphi_{2} & =-\frac{1}{6 \Pi} M_{2} \varphi_{1} \varphi_{1}, \\
\varphi_{3} & =-\frac{1}{6 \Pi} M_{2}\left(\varphi_{2} \varphi_{1}+\varphi_{1} \varphi_{2}\right)-\frac{1}{18 \Pi} M_{3} \varphi_{1} \varphi_{1} \varphi_{1}, \\
\varphi_{4} & =-\frac{1}{6 \Pi} M_{2}\left(\varphi_{2} \varphi_{2}+\varphi_{1} \varphi_{3}+\varphi_{3} \varphi_{1}\right)-\frac{1}{18 \Pi} M_{3}\left(\varphi_{1} \varphi_{1} \varphi_{2}+\varphi_{1} \varphi_{2} \varphi_{1}+\varphi_{2} \varphi_{1} \varphi_{1}\right)-\frac{1}{72 \Pi} M_{4} \varphi_{1} \varphi_{1} \varphi_{1} \varphi_{1} .
\end{aligned}
$$

In Eq. (B1), at leading-order, $\varphi_{1}$ is rewritten with $\delta$. Substituting this relation into the above, we obtain the perturbative expression of $\varphi$ in terms of $\delta$ : 


$$
\begin{aligned}
\varphi_{1}(\boldsymbol{k})= & \frac{\kappa^{2} \rho_{\mathrm{m}}}{3} \frac{\delta(\boldsymbol{k})}{\Pi(k)}, \\
\varphi_{2}(\boldsymbol{k})= & -\frac{1}{6 \Pi(k)}\left(\frac{\kappa^{2} \rho_{\mathrm{m}}}{3}\right)^{2} M_{2}\left(\boldsymbol{k}_{1}, \boldsymbol{k}_{2}\right) \frac{\delta\left(\boldsymbol{k}_{1}\right) \delta\left(\boldsymbol{k}_{2}\right)}{\Pi\left(k_{1}\right) \Pi\left(k_{2}\right)}, \\
\varphi_{3}(\boldsymbol{k})= & -\frac{1}{18 \Pi(k)}\left(\frac{\boldsymbol{\kappa}^{2} \rho_{\mathrm{m}}}{3}\right)^{3}\left[M_{3}\left(\boldsymbol{k}_{1}, \boldsymbol{k}_{2}, \boldsymbol{k}_{3}\right)-\frac{M_{2}\left(\boldsymbol{k}_{12}, \boldsymbol{k}_{3}\right) M_{2}\left(\boldsymbol{k}_{1}, \boldsymbol{k}_{2}\right)}{\Pi\left(k_{12}\right)}\right] \frac{\delta\left(\boldsymbol{k}_{1}\right) \delta\left(\boldsymbol{k}_{2}\right) \delta\left(\boldsymbol{k}_{3}\right)}{\Pi\left(k_{1}\right) \Pi\left(k_{2}\right) \Pi\left(k_{3}\right)} \\
\varphi_{4}(\boldsymbol{k})= & -\frac{1}{72 \Pi(k)}\left(\frac{\kappa^{2} \rho_{\mathrm{m}}}{3}\right)^{4}\left[M_{4}\left(\boldsymbol{k}_{1}, \boldsymbol{k}_{2}, \boldsymbol{k}_{3}, \boldsymbol{k}_{4}\right)+\frac{1}{3} \frac{M_{2}\left(\boldsymbol{k}_{1}, \boldsymbol{k}_{2}\right)}{\Pi\left(k_{12}\right)}\left\{\frac{M_{2}\left(\boldsymbol{k}_{12}, \boldsymbol{k}_{34}\right) M_{2}\left(\boldsymbol{k}_{3}, \boldsymbol{k}_{4}\right)}{\Pi\left(k_{34}\right)}-6 M_{3}\left(\boldsymbol{k}_{12}, \boldsymbol{k}_{3}, \boldsymbol{k}_{4}\right)\right\}\right. \\
& \left.+\frac{2}{3} \frac{M_{2}\left(\boldsymbol{k}_{123}, \boldsymbol{k}_{4}\right)}{\Pi\left(k_{123}\right)} \times\left\{\frac{M_{2}\left(\boldsymbol{k}_{12}, \boldsymbol{k}_{3}\right) M_{2}\left(\boldsymbol{k}_{1}, \boldsymbol{k}_{2}\right)}{\Pi\left(k_{12}\right)}-M_{3}\left(\boldsymbol{k}_{1}, \boldsymbol{k}_{2}, \boldsymbol{k}_{3}\right)\right\}\right] \times \frac{\delta\left(\boldsymbol{k}_{1}\right) \delta\left(\boldsymbol{k}_{2}\right) \delta\left(\boldsymbol{k}_{3}\right) \delta\left(\boldsymbol{k}_{4}\right)}{\Pi\left(k_{1}\right) \Pi\left(k_{2}\right) \Pi\left(k_{3}\right) \Pi\left(k_{4}\right)} .
\end{aligned}
$$

Note that the kernels of integral given above are not yet symmetrized.

Provided the expression $\varphi$ in terms of $\delta$, the vertex functions $\sigma_{21 \cdots 1}$ are now read off from the comparison between Eqs. (4)-(2) and Eq. (7) to give

$$
\sigma^{(n)}\left(\boldsymbol{k}_{1}, \ldots, \boldsymbol{k}_{n}\right)=\frac{1}{2}\left(\frac{k_{12 \cdots n}}{a H}\right)^{2} \times\left(\text { symmetrized kernel of } \varphi_{n}\right),
$$

\section{APPENDIX C: EXPLICIT EXPRESSIONS FOR SUBLEADING CORRECTION IN RESUMMED PROPAGATOR}

In this Appendix, we derive the explicit expressions for the corrections to the propagator arising from the modification of gravity, $\boldsymbol{\delta} G_{a b}$ [Eq. (46)]. To do this, our main task is to calculate the following ensemble average:

$$
\left\langle\Delta^{(n)} \exp \left[\int_{\tau_{0}}^{\tau} d \tau^{\prime \prime} \Xi\left(\boldsymbol{k}, \tau^{\prime \prime}\right)\right]\right\rangle_{\Xi, \omega_{a b}}
$$

Here, the quantity $\sigma^{(n)}$ represents the $n$th order corrections which contribute to the modification of the effective Newton constant via the screening mechanism [see Eq. (36)]. In $f(R)$ gravity, Eq. (37) with the kernels given by Eqs. (A1) and (A2) lead to

$$
\begin{aligned}
\Delta^{(2)}(k ; \tau)= & -\frac{1}{3} \frac{\bar{R}_{, f f}\left(\tau^{\prime}\right)}{\Pi\left(k ; \tau^{\prime}\right)}\left(\frac{\kappa^{2} \rho_{\mathrm{m}}}{3}\right) \int \frac{d^{3} \boldsymbol{p}}{(2 \pi)^{3}} \frac{\delta(\boldsymbol{p} ; \tau)}{\Pi(p ; \tau)} \\
\Delta^{(3)}(k ; \tau)= & -\frac{1}{6} \frac{\bar{R}_{, f f f}\left(\tau^{\prime}\right)}{\Pi\left(k ; \tau^{\prime}\right)}\left(\frac{\kappa^{2} \rho_{\mathrm{m}}}{3}\right)^{2} \int \frac{d^{3} \boldsymbol{p}_{1} d^{3} \boldsymbol{p}_{2}}{(2 \pi)^{6}} \\
& \times\left\{1-\frac{\bar{R}_{, f f}^{2}(\tau)}{3 \bar{R}_{, f f f}(\tau)} \frac{1}{\Pi\left(p_{12} ; \tau\right)}\right\} \\
& \times \frac{\delta\left(\boldsymbol{p}_{1} ; \tau\right) \delta\left(\boldsymbol{p}_{2} ; \tau\right)}{\Pi\left(p_{1} ; \tau\right) \Pi\left(p_{2} ; \tau\right)}
\end{aligned}
$$

Below, based on the linear theory estimate of $\delta$ and $\theta$, we evaluate the nonvanishing contributions to Eq. (C1).

First consider the $n=2$ case in $f(R)$ gravity model. Substituting Eq. (C2) into Eq. (C1), we obtain

$$
\begin{aligned}
\left\langle\Delta^{(2)}\right. & \left.\exp \left[\int_{\tau_{0}}^{\tau} d \tau^{\prime} \Xi\left(\boldsymbol{k}, \tau^{\prime \prime}\right)\right]\right\rangle \\
= & -\frac{1}{3} \frac{\bar{R}_{, f f}\left(\tau^{\prime}\right)}{\Pi\left(k ; \tau^{\prime}\right)}\left(\frac{\kappa^{2} \rho_{\mathrm{m}}}{3}\right)\left\langle\int \frac{d^{3} \boldsymbol{p}}{(2 \pi)^{3}} \frac{\delta\left(\boldsymbol{p} ; \tau^{\prime}\right)}{\Pi\left(p ; \tau^{\prime}\right)}\right. \\
& \left.\times \exp \left[\int_{\tau_{0}}^{\tau} d \tau^{\prime \prime} \int \frac{d^{3} \boldsymbol{q}}{(2 \pi)^{3}}\left(\frac{\boldsymbol{k} \cdot \boldsymbol{q}}{q^{2}}\right) \theta\left(\boldsymbol{q} ; \tau^{\prime \prime}\right)\right]\right\rangle \\
= & -\frac{1}{3} \frac{\bar{R}_{, f f}\left(\tau^{\prime}\right)}{\Pi\left(k ; \tau^{\prime}\right)}\left(\frac{\kappa^{2} \rho_{\mathrm{m}}}{3}\right) \sum_{n=0} \frac{1}{n !} \int \frac{d^{3} \boldsymbol{p} d^{3} \boldsymbol{q}_{1} \cdots d^{3} \boldsymbol{q}_{n}}{(2 \pi)^{3(n+1)}} \\
& \times \int_{\tau_{0}}^{\tau} d \tau_{1}^{\prime \prime} \cdots d \tau_{n}^{\prime \prime}\left(\frac{\boldsymbol{k} \cdot \boldsymbol{q}_{1}}{q_{1}^{2}}\right) \cdots\left(\frac{\boldsymbol{k} \cdot \boldsymbol{q}_{n}}{q_{n}^{2}}\right) \\
& \times\left\langle\frac{\delta\left(\boldsymbol{p} ; \tau^{\prime}\right)}{\Pi\left(p ; \tau^{\prime}\right)} \theta\left(\boldsymbol{q}_{1} ; \tau_{1}^{\prime \prime}\right) \cdots \theta\left(\boldsymbol{q}_{n} ; \tau_{n}^{\prime \prime}\right)\right\rangle \\
= & -\frac{1}{3} \frac{\bar{R}_{, f f}\left(\tau^{\prime}\right)}{\Pi\left(k ; \tau^{\prime}\right)}\left(\frac{\kappa^{2} \rho_{\mathrm{m}}}{3}\right) \int_{\tau_{0}}^{\tau} d \tau^{\prime \prime} \int \frac{d^{3} \boldsymbol{p}}{(2 \pi)^{3}}\left(-\frac{\boldsymbol{k} \cdot \boldsymbol{p}}{p^{2}}\right) \\
& \times \frac{P_{\delta \theta}\left(p ; \tau^{\prime}, \tau^{\prime \prime}\right)}{\Pi\left(p ; \tau^{\prime}\right)} \sum_{m=0} \frac{(2 m+1) ! !}{(2 m+1) !} \\
& \times\left\{-\int_{\tau_{0}}^{\tau} d \tau_{1}^{\prime \prime} d \tau_{2}^{\prime \prime} \int \frac{d^{3} \boldsymbol{q}}{(2 \pi)^{3}}\left(\frac{\boldsymbol{k} \cdot \boldsymbol{q}}{q^{2}}\right)^{2} P_{\theta \theta}\left(p ; \tau_{1}^{\prime \prime}, \tau_{2}^{\prime \prime}\right)\right\}^{m} .
\end{aligned}
$$

Here, in the last line, we have assumed the Gaussianity of the density and velocity-divergence fields. Then, as we see from the integrand, the integral over the mode $\boldsymbol{p}$ becomes vanishing because of the symmetry, and no contribution from $n=2$ can appear in the correction to the high- $k$ limit propagator.

Next focus on the $n=3$ in $f(R)$ gravity, where we can get the nonvanishing correction. Substituting Eq. (C2) into the expression $(\mathrm{C} 1)$, we have 


$$
\begin{aligned}
\left\langle\Delta^{(3)} \exp \left[\int_{\tau_{0}}^{\tau} d \tau^{\prime} \Xi\left(\boldsymbol{k}, \tau^{\prime \prime}\right)\right]\right\rangle= & -\frac{1}{6} \frac{\bar{R}_{, f f f}\left(\tau^{\prime}\right)}{\Pi\left(k ; \tau^{\prime}\right)}\left(\frac{\kappa^{2} \rho_{\mathrm{m}}}{3}\right)^{2} \int \frac{d^{3} \boldsymbol{p}_{1} d^{3} \boldsymbol{p}_{2}}{(2 \pi)^{6}} \times\left\{1-\frac{\bar{R}_{, f f}^{2}(\tau)}{3 \bar{R}_{, f f f}(\tau)} \frac{1}{\Pi\left(p_{12} ; \tau\right)}\right\} \\
& \times\left\langle\frac{\delta\left(\boldsymbol{p}_{1} ; \tau\right) \delta\left(\boldsymbol{p}_{2} ; \tau\right)}{\Pi\left(p_{1} ; \tau\right) \Pi\left(p_{2} ; \tau\right)} \exp \left[\int_{\tau_{0}}^{\tau} d \tau^{\prime \prime} \int \frac{d^{3} \boldsymbol{q}}{(2 \pi)^{3}}\left(\frac{\boldsymbol{k} \cdot \boldsymbol{q}}{q^{2}}\right) \theta\left(\boldsymbol{q} ; \tau^{\prime \prime}\right)\right]\right\rangle \\
= & -\frac{1}{6} \frac{\bar{R}_{, f f f}\left(\tau^{\prime}\right)}{\Pi\left(k ; \tau^{\prime}\right)}\left(\frac{\kappa^{2} \rho_{\mathrm{m}}\left(\tau^{\prime}\right)}{3}\right)^{2} \times\left[\left\{1-\frac{\bar{R}_{, f f}^{2}\left(\tau^{\prime}\right)}{\bar{R}_{, f f f}\left(\tau^{\prime}\right) \bar{R}_{, f}\left(\tau^{\prime}\right)}\right\} \int \frac{d^{3} \boldsymbol{p}}{(2 \pi)^{3}} \frac{P_{\delta \delta}\left(p ; \tau^{\prime}\right)}{\Pi^{2}\left(p ; \tau^{\prime}\right)} \sum_{n=0} \frac{(n-1) ! !}{n !}\right. \\
& \times\left\{-\frac{k^{2}}{3} \int \frac{d q}{2 \pi^{2}}\left\{D_{+}(q ; \tau)-D_{+}\left(q ; \tau_{0}\right)\right\}^{2} P_{0}(q)\right\} \\
& +\int \frac{d^{3} \boldsymbol{p}_{1} d^{3} \boldsymbol{p}_{2}}{(2 \pi)^{6}}\left\{1-\frac{\bar{R}_{, f f}^{2}\left(\tau^{\prime}\right)}{3 \bar{R}_{, f f f}\left(\tau^{\prime}\right)} \frac{1}{\Pi\left(p_{12} ; \tau^{\prime}\right)}\right\}\left(\frac{\boldsymbol{k} \cdot \boldsymbol{p}_{1}}{p_{1}^{2}}\right)\left(\frac{\boldsymbol{k} \cdot \boldsymbol{p}_{2}}{p_{2}^{2}}\right) \frac{P_{0}\left(p_{1}\right) P_{0}\left(p_{2}\right)}{\Pi\left(p_{1} ; \tau^{\prime}\right) \Pi\left(p_{2} ; \tau^{\prime}\right)} \\
& \times D_{+}\left(p_{1} ; \tau^{\prime}\right) D_{+}\left(p_{2} ; \tau^{\prime}\right)\left\{D_{+}\left(p_{1} ; \tau\right)-D_{+}\left(p_{1} ; \tau_{0}\right)\right\}\left\{D_{+}\left(p_{2} ; \tau\right)-D_{+}\left(p_{2} ; \tau_{0}\right)\right\} \\
& \times \sum_{n=0} \frac{(n-3) ! !}{(n-2) !}\left\{-\frac{k^{2}}{3} \int \frac{d q}{2 \pi^{2}}\left\{D_{+}(q ; \tau)-D_{+}\left(q ; \tau_{0}\right)\right\}^{2} P_{0}(q)\right\} \\
= & -\frac{1}{6} \frac{\bar{R}_{, f f f}\left(\tau^{\prime}\right)}{\Pi\left(k ; \tau^{\prime}\right)}\left(\frac{\kappa^{2} \rho_{\mathrm{m}}\left(\tau^{\prime}\right)}{3}\right)^{2} \times\left[1-\frac{\bar{R}_{, f f}^{2}\left(\tau^{\prime}\right)}{\bar{R}_{, f f f}\left(\tau^{\prime}\right) \bar{R}_{, f}\left(\tau^{\prime}\right)}\right\} \int \frac{d p p^{2}}{2 \pi^{2}} \frac{D_{+}^{2}\left(p ; \tau^{\prime}\right) P_{0}(p)}{\Pi^{2}\left(p ; \tau^{\prime}\right)} \\
& +\int \frac{d^{3} \boldsymbol{p}_{1} d^{3} \boldsymbol{p}_{2}}{(2 \pi)^{6}}\left\{1-\frac{\bar{R}_{, f f}^{2}\left(\tau^{\prime}\right)}{3 \bar{R}_{, f f f}\left(\tau^{\prime}\right)} \frac{1}{\Pi\left(p_{12} ; \tau^{\prime}\right)}\right\}\left(\frac{\boldsymbol{k} \cdot \boldsymbol{p}_{1}}{p_{1}^{2}}\right)\left(\frac{\boldsymbol{k} \cdot \boldsymbol{p}_{2}}{p_{2}^{2}}\right) \frac{P_{0}\left(p_{1}\right) P_{0}\left(p_{2}\right)}{\Pi\left(p_{1} ; \tau^{\prime}\right) \Pi\left(p_{2} ; \tau^{\prime}\right)} \\
& \left.\times D_{+}\left(p_{1} ; \tau^{\prime}\right) D_{+}\left(p_{2} ; \tau^{\prime}\right)\left\{D_{+}\left(p_{1} ; \tau\right)-D_{+}\left(p_{1} ; \tau_{0}\right)\right\}\left\{D_{+}\left(p_{2} ; \tau\right)-D_{+}\left(p_{2} ; \tau_{0}\right)\right\}\right] \\
& \times \exp \left[-\frac{k^{2}}{3} \int \frac{d q}{2 \pi^{2}}\left\{D_{+}(q ; \tau)-D_{+}\left(q ; \tau_{0}\right)\right\}^{2} P_{0}(q)\right] .
\end{aligned}
$$

In the above, there appears the integral over the two Fourier modes $\boldsymbol{p}_{1}$ and $\boldsymbol{p}_{2}$, a part of which can be performed analytically. We then have

$$
\begin{aligned}
& \int \frac{d^{3} \boldsymbol{p}_{1} d^{3} \boldsymbol{p}_{2}}{(2 \pi)^{6}}\left\{1-\frac{\bar{R}_{, f f}^{2}\left(\tau^{\prime}\right)}{3 \bar{R}_{, f f}\left(\tau^{\prime}\right)} \frac{1}{\Pi\left(p_{12} ; \tau^{\prime}\right)}\right\}\left(\frac{\boldsymbol{k} \cdot \boldsymbol{p}_{1}}{p_{1}^{2}}\right)\left(\frac{\boldsymbol{k} \cdot \boldsymbol{p}_{2}}{p_{2}^{2}}\right) \frac{P_{0}\left(p_{1}\right) P_{0}\left(p_{2}\right)}{\Pi\left(p_{1} ; \tau^{\prime}\right) \Pi\left(p_{2} ; \tau^{\prime}\right)} \\
& \quad \times D_{+}\left(p_{1} ; \tau^{\prime}\right) D_{+}\left(p_{2} ; \tau^{\prime}\right)\left\{D_{+}\left(p_{1} ; \tau\right)-D_{+}\left(p_{1} ; \tau_{0}\right)\right\}\left\{D_{+}\left(p_{2} ; \tau\right)-D_{+}\left(p_{2} ; \tau_{0}\right)\right\} \\
& =-\frac{k^{2}}{36} \frac{a^{2}\left(\tau^{\prime}\right) \bar{R}_{, f f}^{2}\left(\tau^{\prime}\right)}{\bar{R}_{, f f f}\left(\tau^{\prime}\right)} \int \frac{d p_{1} d p_{2}}{\left(2 \pi^{2}\right)^{2}} \frac{P_{0}\left(p_{1}\right) P_{0}\left(p_{2}\right)}{\Pi\left(p_{1} ; \tau^{\prime}\right) \Pi\left(p_{2} ; \tau^{\prime}\right)} \times D_{+}\left(p_{1} ; \tau^{\prime}\right) D_{+}\left(p_{2} ; \tau^{\prime}\right)\left\{D_{+}\left(p_{1} ; \tau\right)-D_{+}\left(p_{1} ; \tau_{0}\right)\right\} \\
& \quad \times\left\{D_{+}\left(p_{2} ; \tau\right)-D_{+}\left(p_{2} ; \tau_{0}\right)\right\} \times\left\{2-\frac{\bar{R}_{, f}\left(\tau^{\prime}\right) / 3+\left(p_{1}^{2}+p_{2}^{2}\right) / a^{2}\left(\tau^{\prime}\right)}{2 p_{1} p_{2} / a^{2}\left(\tau^{\prime}\right)} \log \left[\frac{\bar{R}_{, f}\left(\tau^{\prime}\right) / 3+\left(p_{1}+p_{2}\right)^{2} / a^{2}\left(\tau^{\prime}\right)}{\bar{R}_{, f}\left(\tau^{\prime}\right) / 3+\left(p_{1}-p_{2}\right)^{2} / a^{2}\left(\tau^{\prime}\right)}\right]\right\} .
\end{aligned}
$$

With the above expression and Eq. (C5), we now obtain the leading-order nonvanishing corrections to the propagator, and the final form of the correction, $\delta g_{a b}^{(3)}$, defined in Eq. (47), becomes

$$
\begin{aligned}
\delta g_{a b}^{(3)}\left(k ; \tau, \tau_{0}\right)= & \int_{\tau_{0}}^{\tau} d \tau^{\prime} g_{a 2}\left(k ; \tau, \tau^{\prime}\right) g_{1 b}\left(k ; \tau^{\prime}, \tau_{0}\right) \frac{\kappa^{2}}{2} \frac{\rho_{\mathrm{m}}\left(\tau^{\prime}\right)}{H^{2}\left(\tau^{\prime}\right)} \frac{1}{3} \frac{(k / a)^{2}}{\Pi\left(k ; \tau^{\prime}\right)}\left(-\frac{1}{6}\right) \frac{\bar{R}_{, f f f}\left(\tau^{\prime}\right)}{\Pi\left(k ; \tau^{\prime}\right)}\left(\frac{\kappa^{2} \rho_{\mathrm{m}}\left(\tau^{\prime}\right)}{3}\right)^{2} \\
& \times\left[\left\{1-\frac{\bar{R}_{, f f}^{2}\left(\tau^{\prime}\right)}{\bar{R}_{, f f f}\left(\tau^{\prime}\right) \bar{R}_{, f}\left(\tau^{\prime}\right)}\right\} \int \frac{d p p^{2}}{2 \pi^{2}} \frac{D_{+}^{2}\left(p ; \tau^{\prime}\right) P_{0}(p)}{\Pi^{2}\left(p ; \tau^{\prime}\right)}-\frac{k^{2}}{36} \frac{a^{2}\left(\tau^{\prime}\right) \bar{R}_{, f f}^{2}\left(\tau^{\prime}\right)}{\bar{R}_{, f f f}\left(\tau^{\prime}\right)} \int \frac{d p_{1} d p_{2}}{(2 \pi)^{2}} \frac{P_{0}\left(p_{1}\right) P_{0}\left(p_{2}\right)}{\Pi\left(p_{1} ; \tau^{\prime}\right) \Pi\left(p_{2} ; \tau^{\prime}\right)}\right. \\
& \times D_{+}\left(p_{1} ; \tau^{\prime}\right) D_{+}\left(p_{2} ; \tau^{\prime}\right)\left\{D_{+}\left(p_{1} ; \tau\right)-D_{+}\left(p_{1} ; \tau_{0}\right)\right\}\left\{D_{+}\left(p_{2} ; \tau\right)-D_{+}\left(p_{2} ; \tau_{0}\right)\right\} \\
& \left.\times\left\{2-\frac{\bar{R}_{, f}\left(\tau^{\prime}\right) / 3+\left(p_{1}^{2}+p_{2}^{2}\right) / a^{2}\left(\tau^{\prime}\right)}{2 p_{1} p_{2} / a^{2}\left(\tau^{\prime}\right)} \log \left[\frac{\bar{R}_{, f}\left(\tau^{\prime}\right) / 3+\left(p_{1}+p_{2}\right)^{2} / a^{2}\left(\tau^{\prime}\right)}{\bar{R}_{, f}\left(\tau^{\prime}\right) / 3+\left(p_{1}-p_{2}\right)^{2} / a^{2}\left(\tau^{\prime}\right)}\right]\right\}\right]
\end{aligned}
$$

in $f(R)$ gravity model. 
Similarly, in the DGP model, the leading-order nonvanishing correction to the propagator is shown to appear from $n=3$ of Eq. (C1). We here skip the detail of the derivation, and simply present the final result. A straightforward but lengthy calculation leads to

$$
\begin{aligned}
\delta g_{a b}^{(3)}\left(k ; \tau, \tau_{0}\right)= & \int_{\tau_{0}}^{\tau} d \tau^{\prime} g_{a 2}\left(k ; \tau, \tau^{\prime}\right) g_{1 b}\left(k ; \tau^{\prime}, \tau_{0}\right) \frac{\kappa^{2}}{2} \frac{\rho_{\mathrm{m}}\left(\tau^{\prime}\right)}{H^{2}\left(\tau^{\prime}\right)} \frac{1}{3} \frac{(k / a)^{2}}{\Pi\left(k ; \tau^{\prime}\right)} \cdot(-3)\left(\frac{r_{c}^{2}}{3 \beta\left(\tau^{\prime}\right)^{2}}\right)^{2}\left(\frac{\kappa^{2} \rho_{\mathrm{m}}\left(\tau^{\prime}\right)}{3}\right)^{2} \\
& \times\left[\frac{32}{45} D_{+}^{2}\left(\tau^{\prime}\right) \int \frac{d p p^{2}}{2 \pi^{2}} P_{0}(p)+\left(D_{+}\left(\tau^{\prime}\right)\left\{D_{+}(\tau)-D_{*}\left(\tau_{0}\right)\right\}\right)^{2} \frac{k^{2}}{3} \int \frac{d p_{1} d p_{2}}{(2 \pi)^{2}} P_{0}\left(p_{1}\right) P_{0}\left(p_{2}\right)\right. \\
& \left.\times\left\{-\frac{2}{45}\left(p_{1}^{2}+p_{2}^{2}\right)+\frac{\left(p_{1}^{2}-p_{2}^{2}\right)\left(p_{1}^{2}+p_{2}^{2}\right)}{60\left(p_{1} p_{2}\right)^{2}}-\frac{\left(p_{1}^{2}-p_{2}^{2}\right)^{4}}{120\left(p_{1} p_{2}\right)^{3}} \log \left|\frac{p_{1}+p_{2}}{p_{1}-p_{2}}\right|\right\}\right] .
\end{aligned}
$$

[1] S. Perlmutter et al. (Supernova Cosmology Project), Astrophys. J. 517, 565 (1999).

[2] A. G. Riess et al. (Supernova Search Team), Astron. J. 116, 1009 (1998).

[3] D. H. Weinberg, M. J. Mortonson, D. J. Eisenstein, C. Hirata, A. G. Riess, and E. Rozo, Phys. Rep. 530, 87 (2013).

[4] C. Alcock and B. Paczynski, Nature (London) 281, 358 (1979).

[5] H.-J. Seo and D. J. Eisenstein, Astrophys. J. 598, 720 (2003).

[6] C. Blake and K. Glazebrook, Astrophys. J. 594, 665 (2003).

[7] K. Glazebrook and C. Blake, Astrophys. J. 631, 1 (2005).

[8] M. Shoji, D. Jeong, and E. Komatsu, Astrophys. J. 693, 1404 (2009).

[9] N. Padmanabhan and M. J. White, Phys. Rev. D 77, 123540 (2008).

[10] N. Kaiser, Mon. Not. R. Astron. Soc. 227, 1 (1987).

[11] A. J. S. Hamilton, arXiv:astro-ph/9708102.

[12] P. Peebles, The Large-Scale Structure of the Universe (Princeton University Press, Princeton, NJ, 1980).

[13] E. V. Linder, Astropart. Phys. 29, 336 (2008).

[14] D. J. Eisenstein et al. (SDSS), Astrophys. J. 633, 560 (2005).

[15] W. J. Percival, S. Cole, D. J. Eisenstein, R. C. Nichol, J. A. Peacock, A. C. Pope, and A. S. Szalay, Mon. Not. R. Astron. Soc. 381, 1053 (2007).

[16] B. A. Reid, L. Samushia, M. White, W. J. Percival, M. Manera, N. Padmanabhan, A. J. Ross, A. G. Sánchez, S. Bailey, D. Bizyaev et al., Mon. Not. R. Astron. Soc. 426, 2719 (2012).

[17] L. Anderson et al. (BOSS Collaboration), Mon. Not. R. Astron. Soc. 441, 24 (2014).

[18] F. Beutler et al. (BOSS Collaboration), arXiv:1312.4611.

[19] D. Jeong and E. Komatsu, Astrophys. J. 651, 619 (2006).

[20] M. Crocce and R. Scoccimarro, Phys. Rev. D 77, 023533 (2008).

[21] T. Matsubara, Phys. Rev. D 77, 063530 (2008).

[22] T. Matsubara, Phys. Rev. D 83, 083518 (2011).

[23] P. McDonald, Phys. Rev. D 75, 043514 (2007).
[24] A. Taruya and T. Hiramatsu, Astrophys. J. 674, 617 (2008).

[25] A. Taruya, T. Nishimichi, S. Saito, and T. Hiramatsu, Phys. Rev. D 80, 123503 (2009).

[26] F. Bernardeau, M. Crocce, and R. Scoccimarro, Phys. Rev. D 78, 103521 (2008).

[27] M. Pietroni, J. Cosmol. Astropart. Phys. 10 (2008) 036.

[28] P. Valageas, Astron. Astrophys. 465, 725 (2007).

[29] A. Taruya, F. Bernardeau, T. Nishimichi, and S. Codis, Phys. Rev. D 86, 103528 (2012).

[30] L. Wang, B. Reid, and M. White, arXiv:1306.1804.

[31] A. Taruya, T. Nishimichi, and S. Saito, Phys. Rev. D 82, 063522 (2010).

[32] B. A. Reid and M. White, Mon. Not. R. Astron. Soc. 417, 1913 (2011).

[33] J. Carlson, B. Reid, and M. White, arXiv:1209.0780.

[34] U. Seljak and P. McDonald, J. Cosmol. Astropart. Phys. 11 (2011) 039.

[35] Z. Vlah, U. Seljak, P. McDonald, T. Okumura, and T. Baldauf, J. Cosmol. Astropart. Phys. 11 (2012) 009.

[36] T. Okumura, U. Seljak, P. McDonald, and V. Desjacques, J. Cosmol. Astropart. Phys. 02 (2012) 010.

[37] K. Heitmann, D. Higdon, M. White, S. Habib, B. J. Williams, E. Lawrence, and C. Wagner, Astrophys. J. 705, 156 (2009).

[38] E. Lawrence, K. Heitmann, M. White, D. Higdon, C. Wagner, S. Habib, and B. Williams, Astrophys. J. 713, 1322 (2010).

[39] W. Hu and I. Sawicki, Phys. Rev. D 76, 104043 (2007).

[40] T. Baker, P. G. Ferreira, and C. Skordis, Phys. Rev. D 87, 024015 (2013).

[41] P. Brax, A.-C. Davis, B. Li, and H. A. Winther, Phys. Rev. D 86, 044015 (2012).

[42] S. F. Daniel, R. R. Caldwell, A. Cooray, and A. Melchiorri, Phys. Rev. D 77, 103513 (2008).

[43] T. Clifton, P. G. Ferreira, A. Padilla, and C. Skordis, Phys. Rep. 513, 1 (2012).

[44] A. Taruya, K. Koyama, T. Hiramatsu, and A. Oka, Phys. Rev. D 89, 043509 (2014). 
[45] K. Koyama, A. Taruya, and T. Hiramatsu, Phys. Rev. D 79, 123512 (2009).

[46] F. Bernardeau, S. Colombi, E. Gaztanaga, and R. Scoccimarro, Phys. Rep. 367, 1 (2002).

[47] P. Brax and P. Valageas, Phys. Rev. D 88, 023527 (2013).

[48] P. Brax and P. Valageas, Phys. Rev. D 86, 063512 (2012).

[49] M. Crocce and R. Scoccimarro, Phys. Rev. D 73, 063519 (2006).

[50] J. Carlson, M. White, and N. Padmanabhan, Phys. Rev. D 80, 043531 (2009).

[51] F. Bernardeau, M. Crocce, and R. Scoccimarro, Phys. Rev. D 85, 123519 (2012).

[52] F. Bernardeau, A. Taruya, and T. Nishimichi, Phys. Rev. D 89, 023502 (2014).

[53] M. Crocce, R. Scoccimarro, and F. Bernardeau, Mon. Not. R. Astron. Soc. 427, 2537 (2012).

[54] A. Taruya, T. Nishimichi, and F. Bernardeau, Phys. Rev. D 87, 083509 (2013).

[55] J. Khoury and A. Weltman, Phys. Rev. D 69, 044026 (2004).

[56] C. Deffayet, G. Dvali, G. Gabadadze, and A. I. Vainshtein, Phys. Rev. D 65, 044026 (2002).

[57] W. Hu and I. Sawicki, Phys. Rev. D 76, 064004 (2007).

[58] A. A. Starobinsky, JETP Lett. 86, 157 (2007).

[59] G. R. Dvali, G. Gabadadze, and M. Porrati, Phys. Lett. B 485, 208 (2000).

[60] A. Marchini and V. Salvatelli, Phys. Rev. D 88, 027502 (2013).

[61] L. Lombriser, A. Slosar, U. Seljak, and W. Hu, Phys. Rev. D 85, 124038 (2012).

[62] K. Yamamoto, G. Nakamura, G. Hutsi, T. Narikawa, and T. Sato, Phys. Rev. D 81, 103517 (2010).

[63] H. Okada, T. Totani, and S. Tsujikawa, Phys. Rev. D 87, 103002 (2013).

[64] F. Schmidt, A. Vikhlinin, and W. Hu, Phys. Rev. D 80, 083505 (2009).

[65] J. Wang, L. Hui, and J. Khoury, Phys. Rev. Lett. 109, 241301 (2012).

[66] F. Bernardeau, N. Van de Rijt, and F. Vernizzi, Phys. Rev. D 85, 063509 (2012).

[67] R. Scoccimarro, Phys. Rev. D 80, 104006 (2009).

[68] K. C. Chan and R. Scoccimarro, Phys. Rev. D 80, 104005 (2009).

[69] B. Falck, K. Koyama, G.-b. Zhao, and B. Li, J. Cosmol. Astropart. Phys. 07 (2014) 058.

[70] T. Hiramatsu and A. Taruya, Phys. Rev. D 79, 103526 (2009).
[71] The numerical PT scheme in [70] solves the moment equations coupled with the propagator $G_{a b}$. For the perturbative treatment, this scheme reproduces the standard PT results at one-loop order. From the standard PT result of $G_{a b}$, we can obtain $\bar{\Gamma}_{a, 1 \text {-loop }}^{(1)}$ through the relation $\left(G_{a b}-g_{a b}\right) u_{b}=\bar{\Gamma}_{a, 1 \text {-loop }}^{(1)}$, where $g_{a b}$ is the linear propagator and $u_{a}=(1,1)$.

[72] B. Li, G.-B. Zhao, R. Teyssier, and K. Koyama, J. Cosmol. Astropart. Phys. 01 (2012) 051.

[73] R. Teyssier, Astron. Astrophys. 385, 337 (2002).

[74] G. Hinshaw et al. (WMAP), Astrophys. J. Suppl. Ser. 208, 19 (2013).

[75] S. Prunet, C. Pichon, D. Aubert, D. Pogosyan, R. Teyssier, and S. Gottloeber, Astrophys. J. Suppl. Ser. 178, 179 (2008).

[76] M. Crocce and R. Scoccimarro, Phys. Rev. D 73, 063520 (2006).

[77] M. Crocce, S. Pueblas, and R. Scoccimarro, Mon. Not. R. Astron. Soc. 373, 369 (2006).

[78] D. J. Eisenstein, H.-j. Seo, and M. J. White, Astrophys. J. 664, 660 (2007).

[79] H.-J. Seo and D. J. Eisenstein, Astrophys. J. 633, 575 (2005).

[80] T. Nishimichi et al., Publ. Astron. Soc. Jpn. 61, 321 (2009).

[81] R. E. Smith, R. Scoccimarro, and R. K. Sheth, Phys. Rev. D 77, 043525 (2008).

[82] R. Scoccimarro, Phys. Rev. D 70, 083007 (2004).

[83] T. Nishimichi and A. Taruya, Phys. Rev. D 84, 043526 (2011).

[84] T. Ishikawa, T. Totani, T. Nishimichi, R. Takahashi, N. Yoshida, and M. Tonegawa, Mon. Not. R. Astron. Soc. 443, 3359 (2014).

[85] A. Oka, S. Saito, T. Nishimichi, A. Taruya, and K. Yamamoto, Mon. Not. R. Astron. Soc. 439, 2515 (2014).

[86] Y.-S. Song, T. Okumura, and A. Taruya, Phys. Rev. D 89, 103541 (2014).

[87] E. V. Linder, M. Oh, T. Okumura, C. G. Sabiu, and Y.-S. Song, Phys. Rev. D 89, 063525 (2014).

[88] E. Jennings, C. M. Baugh, B. Li, G.-B. Zhao, and K. Koyama, Mon. Not. R. Astron. Soc. 425, 2128 (2012).

[89] F. Schmidt, A. Vikhlinin, and W. Hu, Phys. Rev. D 80, 083505 (2009).

[90] F. Schmidt, M. V. Lima, H. Oyaizu, and W. Hu, Phys. Rev. D 79, 083518 (2009).

[91] V. Desjacques and R. K. Sheth, Phys. Rev. D 81, 023526 (2010).

[92] T. Baldauf, V. Desjacques, and U. Seljak, arXiv:1405.5885. 